\title{
Properties of certain new special polynomials associated with Sheffer sequences
}

\author{
Nusrat Raza ${ }^{1}$, Subuhi Khan ${ }^{2}$ and Mahvish $\mathrm{Ali}^{3 *}$ \\ ${ }^{1}$ Women's College, Aligarh Muslim University, Aligarh, India. \\ ${ }^{2,3}$ Department of Mathematics, Aligarh Muslim University, Aligarh, India. \\ ${ }^{2}$ Corresponding author. \\ E-mail: nraza.maths@gmail.com ${ }^{1}$, subuhi2006@gmail. com $^{2}$, mahvishali37@gmail. com $^{3}$
}

\begin{abstract}
In this article, the Laguerre-Gould Hopper polynomials are combined with Sheffer sequences to introduce certain mixed type special polynomials. Certain important properties of these polynomials are established. Further, operational and integral representations for these mixed polynomials are derived.
\end{abstract}

2010 Mathematics Subject Classification. 33C45. 33C99, 33E20

Keywords. Laguerre-Gould Hopper based Sheffer polynomials; Monomiality principle; Operational techniques.

\section{Introduction and preliminaries}

We recall that the polynomial sequence $\left\{s_{n}(x)\right\}_{n=0}^{\infty}\left(s_{n}(x)\right.$ being a polynomial of degree $\left.n\right)$ is called Sheffer A-type zero [39, p.222 (Theorem 72)], (which we shall hereafter call Sheffer-type), if $s_{n}(x)$ possesses the exponential generating function of the form

$$
A(t) \exp (x H(t))=\sum_{n=0}^{\infty} s_{n}(x) \frac{t^{n}}{n !},
$$

where $A(t)$ and $H(t)$ have (at least the formal) expansions:

$$
A(t)=\sum_{n=0}^{\infty} A_{n} \frac{t^{n}}{n !}, \quad A_{0} \neq 0
$$

and

$$
H(t)=\sum_{n=1}^{\infty} H_{n} \frac{t^{n}}{n !}, \quad H_{1} \neq 0
$$

respectively.

Also, in view of the following result [40, p.17], the Sheffer sequences can be alternatively defined as:

*This work has been done under Junior Research Fellowship (Award letter No. F1-17.1/2014-15/ MANF-2014-15MUS-UTT-34170/(SA-III/Website)) awarded to the third author by the University Grants Commission, Government of India, New Delhi. 
Let $f(t)$ be a delta series and let $g(t)$ be an invertible series of the following form:

$$
f(t)=\sum_{n=0}^{\infty} f_{n} \frac{t^{n}}{n !}, \quad f_{0}=0, f_{1} \neq 0
$$

and

$$
g(t)=\sum_{n=0}^{\infty} g_{n} \frac{t^{n}}{n !}, \quad g_{0} \neq 0 .
$$

Then there exists a unique sequence $s_{n}(x)$ of polynomials satisfying the orthogonality conditions

$$
\left\langle g(t) f(t)^{k} \mid s_{n}(x)\right\rangle=n ! \delta_{n, k}, \quad \forall n, k \geq 0 .
$$

According to Roman [40, p.18 (Theorem 2.3.4)], the polynomial sequence $s_{n}(x)$ is uniquely determined by two (formal) power series given by equations (1.3a) and (1.3b). The exponential generating function of $s_{n}(x)$ is then given by

$$
\frac{1}{g\left(f^{-1}(t)\right)} \exp \left(x f^{-1}(t)\right)=\sum_{n=0}^{\infty} s_{n}(x) \frac{t^{n}}{n !},
$$

for all $x$ in $\mathbb{C}$, where $f^{-1}(t)$ is the compositional inverse of $f(t)$.

In view of equations (1.1) and (1.5), we have

$$
A(t)=\frac{1}{g\left(f^{-1}(t)\right)}
$$

and

$$
H(t)=f^{-1}(t)
$$

The sequence $s_{n}(x)$ in equation (1.4) is the Sheffer sequence for the pair $(g(t), f(t))$. The Sheffer sequence for $(1, f(t))$ is called the associated Sheffer sequence for $f(t)$ and the Sheffer sequence for $(g(t), t)$ becomes the Appell sequence for $g(t)[40$, p. 17]. Properties of Appell and Sheffer sequences are naturally handled within the framework of modern classical umbral calculus by Ro$\operatorname{man}[40]$.

The idea of monomiality arised within the context of poweroid, suggested by J. F. Steffenson [41]. The monomiality principle has been proved to be a powerful tool for the investigation of the properties of a wide class of polynomials like Sheffer polynomials, see for example [15]. The monomiality principle is reformulated and developed by Dattoli [9], according to which, the polynomial set $\left\{p_{n}(x)\right\}_{n \in \mathbb{N}}$ is "quasi-monomial", provided there exist two operators $\hat{M}$ and $\hat{P}$ playing, respectively, the role of multiplicative and derivative operators, for the family of polynomials. These operators satisfy the following identities:

$$
\hat{M}\left\{p_{n}(x)\right\}=p_{n+1}(x)
$$

and

$$
\hat{P}\left\{p_{n}(x)\right\}=n p_{n-1}(x),
$$


for all $n \in \mathbb{N}$. The operators $\hat{M}$ and $\hat{P}$ also satisfy the commutation relation

$$
[\hat{P}, \hat{M}]=\hat{P} \hat{M}-\hat{M} \hat{P}=\hat{1}
$$

and thus display the Weyl group structure. If the considered polynomial set $\left\{p_{n}(x)\right\}_{n \in \mathbb{N}}$ is quasimonomial, its properties can easily be derived from those of the $\hat{M}$ and $\hat{P}$ operators. In fact:

(i) Combining recurrences (1.7) and (1.8), we have

$$
\hat{M} \hat{P}\left\{p_{n}(x)\right\}=n p_{n}(x),
$$

which can be interpreted as the differential equation satisfied by $p_{n}(x)$, if $\hat{M}$ and $\hat{P}$ have a differential realization.

(ii) Assuming here and in the sequel $p_{0}(x)=1$, then $p_{n}(x)$ can be explicitly constructed as:

$$
p_{n}(x)=\hat{M}^{n}\{1\},
$$

which yields the series definition for $p_{n}(x)$.

(iii) Identity (1.11) implies that the exponential generating function of $p_{n}(x)$ can be given in the form:

$$
\exp (t \hat{M})\{1\}=\sum_{n=0}^{\infty} p_{n}(x) \frac{t^{n}}{n !}, \quad|t|<\infty .
$$

We recall that the Laguerre-Gould Hopper polynomials (LGHP) ${ }_{L} H_{n}^{(m, r)}(x, y, z)$ are introduced in [29] and defined by the following generating function:

$$
C_{0}\left(-x t^{m}\right) \exp \left(y t+z t^{r}\right)=\sum_{n=0}^{\infty}{ }_{L} H_{n}^{(m, r)}(x, y, z) \frac{t^{n}}{n !},
$$

where $C_{0}(x)$ denotes the Bessel-Tricomi function of order 0. The $n t h$ order Bessel-Tricomi function $C_{n}(x)$ defined by the following series [9, p.150]:

$$
C_{n}(x)=x^{-\frac{n}{2}} J_{n}(2 \sqrt{x})=\sum_{k=0}^{\infty} \frac{(-1)^{k} x^{k}}{k !(n+k) !}, \quad n=0,1,2, \ldots,
$$

with $J_{n}(x)$ being the ordinary cylindrical Bessel function of first kind [1]. The $0^{\text {th }}$-order BesselTricomi function $C_{0}(x)$ is also given by the following operational definition:

$$
C_{0}(\alpha x)=\exp \left(-\alpha D_{x}^{-1}\right)\{1\}
$$

where $D_{x}^{-1}$ denotes the inverse derivative operator and

$$
D_{x}^{-n}\{1\}=\frac{x^{n}}{n !} .
$$


The LGHP ${ }_{L} H_{n}^{(m, r)}(x, y, z)$ are shown to be quasi-monomial under the action of the following multiplicative and derivative operators [29]:

$$
\hat{M}_{L H}:=y+m D_{x}^{-1} \frac{\partial^{m-1}}{\partial y^{m-1}}+r z \frac{\partial^{r-1}}{\partial y^{r-1}}
$$

and

$$
\hat{P}_{L H}:=\frac{\partial}{\partial y}
$$

respectively.

The LGHP ${ }_{L} H_{n}^{(m, r)}(x, y, z)$ are important as they contain several special polynomials as their particular cases. For suitable values of the indices and variables the $\operatorname{LGHP}_{L} H_{n}^{(m, r)}(x, y, z)$ reduce to these special polynomials. We mention these special cases in the following table:

\begin{tabular}{|c|c|c|c|c|}
\hline $\begin{array}{l}\text { S. } \\
\text { No. }\end{array}$ & $\begin{array}{l}\text { Values of } \\
\text { the Indices } \\
\text { and Variables }\end{array}$ & $\begin{array}{l}\text { Relation Between the } \\
\text { LGHP } L_{n}^{(m, s)}(x, y, z) \\
\text { and its Special Case }\end{array}$ & $\begin{array}{l}\text { Name of } \\
\text { the Known } \\
\text { Polynomials }\end{array}$ & $\begin{array}{l}\text { Generating Functions of } \\
\text { the Known Polynomials }\end{array}$ \\
\hline I. & $\begin{array}{l}m=1, \quad r=2 \\
x \rightarrow-x\end{array}$ & ${ }_{L} H_{n}^{(1,2)}(-x, y, z)={ }_{L} H_{n}(x, y, z)$ & $\begin{array}{l}\text { 3-Variable } \\
\text { Laguerre - } \\
\text { Hermite [23] }\end{array}$ & $C_{0}(x t) \exp \left(y t+z t^{2}\right)=\sum_{n=0}^{\infty} L_{n} H_{n}(x, y, z) \frac{t^{n}}{n !}$ \\
\hline II. & $\begin{aligned} m & =1, r=2 \\
z & =-\frac{1}{2} \\
x & \rightarrow-x\end{aligned}$ & ${ }_{L} H_{n}^{(1,2)}\left(-x, y,-\frac{1}{2}\right)={ }_{L} H_{n}^{*}(x, y)$ & $\begin{array}{l}\text { 2-Variable } \\
\text { Laguerre - } \\
\text { Hermite [24] }\end{array}$ & $C_{0}(x t) \exp \left(y t-\frac{1}{2} t^{2}\right)=\sum_{n=0}^{\infty} L_{n}^{*}(x, y) \frac{t^{n}}{n !}$ \\
\hline III. & $\begin{aligned} m & =1, r=2 \\
y & =1, z \rightarrow y \\
x & \rightarrow-x\end{aligned}$ & $L_{n}^{(1,2)}(-x, 1, y)=\varphi_{n}(x, y)$ & $\begin{array}{l}\text { Laguerre- } \\
\text { Hermite } \\
\text { type }[22]\end{array}$ & $C_{0}(x t) \exp \left(t+y t^{2}\right)=\sum_{n=0}^{\infty} \varphi_{n}(x, y) \frac{t^{n}}{n !}$ \\
\hline IV. & $x=0$ & ${ }_{L} H_{n}^{(m, r)}(0, y, z)=H_{n}^{(r)}(y, z)$ & $\begin{array}{l}\text { Gould- } \\
\text { Hopper [27] }\end{array}$ & $\exp \left(y t+z t^{r}\right)=\sum_{n=0}^{\infty} H_{n}^{(r)}(y, z) \frac{t^{n}}{n !}$ \\
\hline V. & $z=0$ & ${ }_{L} H_{n}^{(m, r)}(x, y, 0)={ }_{m} L_{n}(x, y)$ & $\begin{array}{l}\text {-Variable } \\
\text { generalized } \\
\text { Laguerre [13] }\end{array}$ & $C_{0}\left(-x t^{m}\right) \exp (y t)=\sum_{n=0}^{\infty} m L_{n}(x, y) \frac{t^{n}}{n !}$ \\
\hline VI. & $\begin{array}{l}r=m ; x=0 \\
y \rightarrow-D_{x}^{-1} \\
z \rightarrow y\end{array}$ & $\begin{array}{l}L_{n}^{(m, m)}\left(0,-D_{x}^{-1}, y\right) \\
={ }_{[m]} L_{n}(x, y)\end{array}$ & $\begin{array}{l}\text { 2-Variable } \\
\text { generalized } \\
\text { Laguerre } \\
\text { type }[11]\end{array}$ & $C_{0}(x t) \exp \left(y t^{m}\right)=\sum_{n=0[m]}^{\infty} L_{n}(x, y) \frac{t^{n}}{n !}$ \\
\hline VII. & $\begin{array}{l}r=m-1 ; x=0 \\
y \rightarrow x, z \rightarrow y\end{array}$ & ${ }_{L} H_{n}^{(m, m-1)}(0, x, y)=U_{n}^{(m)}(x, y)$ & $\begin{array}{l}\text { Generalized } \\
\text { Chebyshev [10] }\end{array}$ & $\exp \left(x t+y t^{m-1}\right)=\sum_{n=0}^{\infty} U_{n}^{(m)}(x, y) \frac{t^{n}}{n !}$ \\
\hline VIII. & $\begin{array}{l}m=1 ; z=0 \\
x \rightarrow-x\end{array}$ & $L_{n} H_{n}^{(1, r)}(-x, y, 0)=L_{n}(x, y)$ & $\begin{array}{l}\text { 2-Variable } \\
\text { Laguerre }[20]\end{array}$ & $C_{0}(x t) \exp (y t)=\sum_{n=0}^{\infty} L_{n}(x, y) \frac{t^{n}}{n !}$ \\
\hline IX. & $\begin{array}{l}m=1 ; z=0 \\
x \rightarrow y \\
y \rightarrow-D_{x}^{-1}\end{array}$ & $\begin{array}{l}n !_{L} H_{n}^{(1, r)}\left(y,-D_{x}^{-1}, 0\right) \\
=R_{n}(x, y)\end{array}$ & $\begin{array}{l}\text { 2-Variable } \\
\text { Legendre }[17]\end{array}$ & $C_{0}(x t) C_{0}(-y t)=\sum_{n=0}^{\infty} \frac{R_{n}(x, y)}{n !} \frac{t^{n}}{n !}$ \\
\hline $\mathrm{X}$. & $\begin{array}{l}x=0, y \rightarrow x \\
z \rightarrow y D_{y} y\end{array}$ & $L H_{n}^{(m, r)}\left(0, x, y D_{y} y\right)=e_{n}^{(r)}(x, y)$ & $\begin{array}{l}\text { 2-Variable } \\
\text { truncated of } \\
\text { order } s[11] \\
(\text { see }[14])\end{array}$ & $\frac{1}{\left(1-y t^{r}\right)} \exp (x t)=\sum_{n=0}^{\infty} e_{n}^{(r)}(x, y) \frac{t^{n}}{n !}$ \\
\hline XI. & $r=2 ; x=0$ & ${ }_{L} H_{n}^{(m, 2)}(0, y, z)=H_{n}(y, z)$ & $\begin{array}{l}\text { 2-Variable } \\
\text { Hermite-Kampé } \\
\text { de Fériet [3] }\end{array}$ & $\exp \left(y t+z t^{2}\right)=\sum_{n=0}^{\infty} H_{n}(y, z) \frac{t^{n}}{n !}$ \\
\hline XII. & $\begin{array}{l}r=2 ; x=0 \\
y \rightarrow D_{x}^{-1}, z \rightarrow y\end{array}$ & $\begin{array}{l}L_{n}^{(m, 2)}\left(0, D_{x}^{-1}, y\right) \\
=G_{n}(x, y)\end{array}$ & $\begin{array}{l}\text { Hermite } \\
\text { type [12] }\end{array}$ & $C_{0}(-x t) \exp \left(y t^{2}\right)=\sum_{n=0}^{\infty} G_{n}(x, y) \frac{t^{n}}{n !}$ \\
\hline XIII. & $\begin{array}{l}m=2 ; z=0 \\
x \rightarrow\left(\frac{x^{2}-1}{4}\right) \\
y \rightarrow x\end{array}$ & ${ }_{L} H_{n}^{(2, r)}\left(\frac{x^{2}-1}{4}, x, 0\right)=P_{n}(x)$ & Legendre [39] & $C_{0}\left(-\frac{\left(x^{2}-1\right)}{4} t^{2}\right) \exp (x t)=\sum_{n=0}^{\infty} P_{n}(x) \frac{t^{n}}{n !}$ \\
\hline XIV. & $\begin{array}{l}x \rightarrow y D y y \\
y \rightarrow x\end{array}$ & $\begin{array}{l}L^{H_{n}^{(m, r)}}\left(y D_{y} y, x, z\right) \\
=H_{n}^{(r, m)}(x, y, z)\end{array}$ & $\begin{array}{l}\text { 3-Variable } \\
\text { generalized } \\
\text { Hermite [18] }\end{array}$ & $\exp \left(x t+y t^{m}+z t^{r}\right)=\sum_{n=0}^{\infty} H_{n}^{(r, m)}(x, y, z) \frac{t^{n}}{n !}$ \\
\hline $\mathrm{XV}$. & $\begin{array}{l}m=2, r=3 \\
x \rightarrow z D_{z} z \\
y \rightarrow x, z \rightarrow y\end{array}$ & $\begin{array}{l}L_{n}^{H_{n}^{(2,3)}\left(z D_{z} z, x, y\right)} \\
=H_{n}^{(3,2)}(x, y, z)\end{array}$ & Bell-type [21] & $\exp \left(x t+y t^{3}+z t^{2}\right)=\sum_{n=0}^{\infty} H_{n}^{(3,2)}(x, y, z) \frac{t^{n}}{n !}$ \\
\hline
\end{tabular}

Table 1. Special cases of the LGHP ${ }_{L} H_{n}^{(m, r)}(x, y, z)$ 
The correspondence given in Table 1 can be used to derive the results for the polynomials related to the LGHP ${ }_{L} H_{n}^{(m, r)}(x, y, z)$.

In this paper, the families of Laguerre-Gould Hopper based Sheffer polynomials are introduced by using the concepts and the methods associated with monomiality principle. In Section 2 , we introduce the Laguerre-Gould Hopper based Sheffer polynomials (LGHSP) ${ }_{L} H^{(m, r)} s_{n}(x, y, z)$ and frame these polynomials within the context of monomiality principle formalism. In Section 3 , we consider some examples of these polynomials. In Section 4, the operational and integral representations for the Laguerre-Gould Hopper based Sheffer polynomials are established. In Section 5, results are obtained for the members of Laguerre-Gould Hopper based Sheffer and Laguerre-Gould Hopper based associated Sheffer polynomial families by considering some members of the Sheffer and associated Sheffer families respectively.

\section{Laguerre-Gould Hopper based Sheffer polynomials}

To introduce the Laguerre-Gould Hopper based Sheffer polynomials ( LGHSP) denoted by ${ }_{L} H^{(m, r)} s_{n}(x, y, z)$, we prove the following result:

Theorem 2.1. The Laguerre-Gould Hopper based Sheffer polynomials ${ }_{L} H^{(m, r)} s_{n}(x, y, z)$ are defined by the generating function

$$
\frac{1}{g\left(f^{-1}(t)\right)} C_{0}\left(-x\left(f^{-1}(t)\right)^{m}\right) \exp \left(y f^{-1}(t)+z\left(f^{-1}(t)\right)^{r}\right)=\sum_{n=0}^{\infty}{ }_{L} H^{(m, r)} s_{n}(x, y, z) \frac{t^{n}}{n !},
$$

or, equivalently

$$
A(t) C_{0}\left(-x(H(t))^{m}\right) \exp \left(y H(t)+z(H(t))^{r}\right)=\sum_{n=0}^{\infty}{ }_{L} H^{(m, r)} s_{n}(x, y, z) \frac{t^{n}}{n !} .
$$

Proof. Replacing $x$ in the l.h.s. and r.h.s of equation (1.5) by the multiplicative operator $\hat{M}_{L H}$ of the LGHP ${ }_{L} H_{n}^{(m, r)}(x, y, z)$, we have

$$
\frac{1}{g\left(f^{-1}(t)\right)} \exp \left(\hat{M}_{L H} f^{-1}(t)\right)=\sum_{n=0}^{\infty} s_{n}\left(\hat{M}_{L H}\right) \frac{t^{n}}{n !} .
$$

Using the expression of $\hat{M}_{L H}$ given in equation (1.17) and then decoupling the exponential operator in the l.h.s. of the resultant equation by using the Crofton-type identity [16, p. 12]

we find

$$
f\left(y+m \lambda \frac{d^{m-1}}{d y^{m-1}}\right)\{1\}=\exp \left(\lambda \frac{d^{m}}{d y^{m}}\right)\{f(y)\}
$$

$\frac{1}{g\left(f^{-1}(t)\right)} \exp \left(z \frac{\partial^{r}}{\partial y^{r}}\right) \exp \left(\left(y+m D_{x}^{-1} \frac{\partial^{m-1}}{\partial y^{m-1}}\right) f^{-1}(t)\right)=\sum_{n=0}^{\infty} s_{n}\left(y+m D_{x}^{-1} \frac{\partial^{m-1}}{\partial y^{m-1}}+r z \frac{\partial^{r-1}}{\partial y^{r-1}}\right) \frac{t^{n}}{n !}$, 
which on further use of identity (2.4) gives

$$
\frac{1}{g\left(f^{-1}(t)\right)} \exp \left(z \frac{\partial^{r}}{\partial y^{r}}\right) \exp \left(D_{x}^{-1} \frac{\partial^{m}}{\partial y^{m}}\right) \exp \left(y f^{-1}(t)\right)=\sum_{n=0}^{\infty} s_{n}\left(y+m D_{x}^{-1} \frac{\partial^{m-1}}{\partial y^{m-1}}+r z \frac{\partial^{r-1}}{\partial y^{r-1}}\right) \frac{t^{n}}{n !} .
$$

Now, expanding the second exponential in the l.h.s. of equation (2.5) and using definition (1.15), we find

$\frac{1}{g\left(f^{-1}(t)\right)} C_{0}\left(-x\left(f^{-1}(t)\right)^{m}\right) \exp \left(z \frac{\partial^{r}}{\partial y^{r}}\right) \exp \left(y f^{-1}(t)\right)=\sum_{n=0}^{\infty} s_{n}\left(y+m D_{x}^{-1} \frac{\partial^{m-1}}{\partial y^{m-1}}+r z \frac{\partial^{r-1}}{\partial y^{r-1}}\right) \frac{t^{n}}{n !}$.

Again, expanding the first exponential in the l.h.s. of equation (2.6) and denoting the resultant LGHSP in the r.h.s. by ${ }_{L} H^{(m, r)} s_{n}(x, y, z)$, that is

$$
{ }_{L} H^{(m, r)} s_{n}(x, y, z)=s_{n}\left(\hat{M}_{L H}\right)=s_{n}\left(y+m D_{x}^{-1} \frac{\partial^{m-1}}{\partial y^{m-1}}+r z \frac{\partial^{r-1}}{\partial y^{r-1}}\right),
$$

we get assertion (2.1). Also, in view of equations (1.6a) and (1.6b), generating function (2.1) can be expressed equivalently as equation (2.2).

In order to show that the LGHSP ${ }_{L} H^{(m, r)} s_{n}(x, y, z)$ satisfy the monomiality property, we prove the following result:

Theorem 2.2. The Laguerre-Gould Hopper based Sheffer polynomials ${ }_{L} H^{(m, r)} s_{n}(x, y, z)$ are quasimonomial under the action of the following multiplicative and derivative operators:

$$
\hat{M}_{L H s}=\left(y+m D_{x}^{-1} \frac{\partial^{m-1}}{\partial y^{m-1}}+r z \frac{\partial^{r-1}}{\partial y^{r-1}}-\frac{g^{\prime}\left(\partial_{y}\right)}{g\left(\partial_{y}\right)}\right) \frac{1}{f^{\prime}\left(\partial_{y}\right)},
$$

or, equivalently

$$
\hat{M}_{L H s}=\left(y+m D_{x}^{-1} \frac{\partial^{m-1}}{\partial y^{m-1}}+r z \frac{\partial^{r-1}}{\partial y^{r-1}}\right) H^{\prime}\left(H^{-1}\left(\partial_{y}\right)\right)+\frac{A^{\prime}\left(H^{-1}\left(\partial_{y}\right)\right)}{A\left(H^{-1}\left(\partial_{y}\right)\right)}
$$

and

or, equivalently

$$
\hat{P}_{L H s}=f\left(\partial_{y}\right)
$$

$$
\hat{P}_{L H s}=H^{-1}\left(\partial_{y}\right)
$$

respectively, where $\partial_{y}:=\frac{\partial}{\partial y}$.

Proof. Consider the following identity:

$$
\partial_{y}\left\{\exp \left(y f^{-1}(t)+z\left(f^{-1}(t)\right)^{r}\right)\right\}=f^{-1}(t) \exp \left(y f^{-1}(t)+z\left(f^{-1}(t)\right)^{r}\right) .
$$

Since $f^{-1}$ denotes the compositional inverse of the function $f$ and $f(t)$ has an expansion (1.3a) in powers of $t$, therefore we have

$$
f\left(\partial_{y}\right)\left\{\exp \left(y f^{-1}(t)+z\left(f^{-1}(t)\right)^{r}\right)\right\}=t \exp \left(y f^{-1}(t)+z\left(f^{-1}(t)\right)^{r}\right) .
$$


Differentiating equation (2.3) partially with respect to $t$ and in view of relation (2.7), we find

$$
\left(\left(\hat{M}_{L H}-\frac{g^{\prime}\left(f^{-1}(t)\right)}{g\left(f^{-1}(t)\right)}\right) \frac{1}{f^{\prime}\left(f^{-1}(t)\right)}\right) \frac{1}{g\left(f^{-1}(t)\right)} \exp \left(\hat{M}_{L H} f^{-1}(t)\right)=\sum_{n=0}^{\infty}{ }_{L} H^{(m, r)} s_{n+1}(x, y, z) \frac{t^{n}}{n !},
$$

which on using monomiality principle equation (1.12) with $t=f^{-1}(t)$ gives

$$
\begin{gathered}
\left(\left(\hat{M}_{L H}-\frac{g^{\prime}\left(f^{-1}(t)\right)}{g\left(f^{-1}(t)\right)}\right) \frac{1}{f^{\prime}\left(f^{-1}(t)\right)}\right) \frac{1}{g\left(f^{-1}(t)\right)} C_{0}\left(-x\left(f^{-1}(t)\right)^{m}\right) \exp \left(y f^{-1}(t)+z\left(f^{-1}(t)\right)^{r}\right) \\
=\sum_{n=0 L}^{\infty} H^{(m, r)} s_{n+1}(x, y, z) \frac{t^{n}}{n !} .
\end{gathered}
$$

Since $g(t)$ is an invertible series and $f(t)$ is a delta series of $t$ therefore $\frac{g^{\prime}\left(f^{-1}(t)\right)}{g\left(f^{-1}(t)\right)}$ and $\frac{1}{f^{\prime}\left(f^{-1}(t)\right)}$ possess power series expansions of $f^{-1}(t)$. Thus, in view of relation $(2.10)$, the above equation becomes

$$
\begin{gathered}
\left(\left(\hat{M}_{L H}-\frac{g^{\prime}\left(\partial_{y}\right)}{g\left(\partial_{y}\right)}\right) \frac{1}{f^{\prime}\left(\partial_{y}\right)}\right)\left\{\frac{1}{g\left(f^{-1}(t)\right)} C_{0}\left(-x\left(f^{-1}(t)\right)^{m}\right) \exp \left(y f^{-1}(t)+z\left(f^{-1}(t)\right)^{r}\right)\right\} \\
=\sum_{n=0{ }_{L} H^{(m, r)}}^{\infty} s_{n+1}(x, y, z) \frac{t^{n}}{n !},
\end{gathered}
$$

which on using generating function (2.1) becomes

$$
\left(\left(\hat{M}_{L H}-\frac{g^{\prime}\left(\partial_{y}\right)}{g\left(\partial_{y}\right)}\right) \frac{1}{f^{\prime}\left(\partial_{y}\right)}\right)\left\{\sum_{n=0}^{\infty}{ }_{L} H^{(m, r)} s_{n}(x, y, z) \frac{t^{n}}{n !}\right\}=\sum_{n=0}^{\infty}{ }_{L} H^{(m, r)} s_{n+1}(x, y, z) \frac{t^{n}}{n !},
$$

or, equivalently

$$
\sum_{n=0}^{\infty}\left(\left(\hat{M}_{L H}-\frac{g^{\prime}\left(\partial_{y}\right)}{g\left(\partial_{y}\right)}\right) \frac{1}{f^{\prime}\left(\partial_{y}\right)}\right)\left\{_{L} H^{(m, r)} s_{n}(x, y, z)\right\} \frac{t^{n}}{n !}=\sum_{n=0}^{\infty}{ }_{L} H^{(m, r)} s_{n+1}(x, y, z) \frac{t^{n}}{n !} .
$$

Now, equating the coefficients of like powers of $t$ in the above equation, we find

$$
\left(\left(\hat{M}_{L H}-\frac{g^{\prime}\left(\partial_{y}\right)}{g\left(\partial_{y}\right)}\right) \frac{1}{f^{\prime}\left(\partial_{y}\right)}\right)\left\{{ }_{L} H^{(m, r)} s_{n}(x, y, z)\right\}={ }_{L} H^{(m, r)} s_{n+1}(x, y, z),
$$

which, in view of equation (1.7) shows that the multiplicative operator for ${ }_{L} H^{(m, r)} s_{n}(x, y, z)$ is given as:

$$
\hat{M}_{L H s}=\left(\hat{M}_{L H}-\frac{g^{\prime}\left(\partial_{y}\right)}{g\left(\partial_{y}\right)}\right) \frac{1}{f^{\prime}\left(\partial_{y}\right)} .
$$

Finally, using equation (1.17) in the r.h.s of above equation, we get assertion (2.8a).

Again, in view of identity (2.11), we have

$$
\begin{aligned}
f\left(\partial_{y}\right)\left\{\frac{1}{g\left(f^{-1}(t)\right)} C_{0}\left(-x\left(f^{-1}(t)\right)^{m}\right) \exp \left(y f^{-1}(t)+z\left(f^{-1}(t)\right)^{r}\right)\right\} \\
=t \frac{1}{g\left(f^{-1}(t)\right)} C_{0}\left(-x\left(f^{-1}(t)\right)^{m}\right) \exp \left(y f^{-1}(t)+z\left(f^{-1}(t)\right)^{r}\right),
\end{aligned}
$$


which on using generating function (2.1) becomes

$$
f\left(\partial_{y}\right)\left\{\sum_{n=0}^{\infty}{ }_{L} H^{(m, r)} s_{n}(x, y, z) \frac{t^{n}}{n !}\right\}=\sum_{n=1}^{\infty}{ }_{L} H^{(m, r)} s_{n-1}(x, y, z) \frac{t^{n}}{(n-1) !},
$$

or, equivalently

$$
\sum_{n=0}^{\infty} f\left(\partial_{y}\right)\left\{_{L} H^{(m, r)} s_{n}(x, y, z)\right\} \frac{t^{n}}{n !}=\sum_{n=1}^{\infty}{ }_{L} H^{(m, r)} s_{n-1}(x, y, z) \frac{t^{n}}{(n-1) !} .
$$

Equating the coefficients of like powers of $t$ in the above equation, we get

$$
f\left(\partial_{y}\right)\left\{{ }_{L} H^{(m, r)} s_{n}(x, y, z)\right\}=n_{L} H^{(m, r)} s_{n-1}(x, y, z), \quad n \geq 1,
$$

which in view of equation (1.8) yields assertion (2.9a). Also, in view of relations (1.6a) and (1.6b), assertions (2.8a) and (2.9a) can be expressed equivalently as equations (2.8b) and (2.9b), respectively.

Remark 2.1. In view of equation (1.11) and using equations (2.8a) and (2.8b), we deduce the following consequence of Theorem 2.2 .

Corollary 2.1. The Laguerre-Gould Hopper based Sheffer polynomials ${ }_{L} H^{(m, r)} s_{n}(x, y, z)$ have the following explicit representations:

$$
{ }_{L} H^{(m, r)} s_{n}(x, y, z)=\hat{M}_{L H s}^{n}\{1\}
$$

that is,

$$
{ }_{L} H^{(m, r)} s_{n}(x, y, z)=\left(\left(y+m D_{x}^{-1} \frac{\partial^{m-1}}{\partial y^{m-1}}+r z \frac{\partial^{r-1}}{\partial y^{r-1}}-\frac{g^{\prime}\left(\partial_{y}\right)}{g\left(\partial_{y}\right)}\right) \frac{1}{f^{\prime}\left(\partial_{y}\right)}\right)^{n}\{1\},
$$

or, equivalently,

$$
{ }_{L} H^{(m, r)} s_{n}(x, y, z)=\left(\left(y+m D_{x}^{-1} \frac{\partial^{m-1}}{\partial y^{m-1}}+r z \frac{\partial^{r-1}}{\partial y^{r-1}}\right) H^{\prime}\left(H^{-1}\left(\partial_{y}\right)\right)+\frac{A^{\prime}\left(H^{-1}\left(\partial_{y}\right)\right)}{A\left(H^{-1}\left(\partial_{y}\right)\right)}\right)^{n}\{1\} .
$$

Theorem 2.3. The Laguerre-Gould Hopper based Sheffer polynomials ${ }_{L} H^{(m, r)} s_{n}(x, y, z)$ satisfy the following differential equation:

$$
\left(\left(y+m D_{x}^{-1} \frac{\partial^{m-1}}{\partial y^{m-1}}+r z \frac{\partial^{r-1}}{\partial y^{r-1}}-\frac{g^{\prime}\left(\partial_{y}\right)}{g\left(\partial_{y}\right)}\right) \frac{f\left(\partial_{y}\right)}{f^{\prime}\left(\partial_{y}\right)}-n\right)_{L} H^{(m, r)} s_{n}(x, y, z)=0,
$$

or, equivalently

$$
\begin{array}{r}
\left(\left(\left(y+m D_{x}^{-1} \frac{\partial^{m-1}}{\partial y^{m-1}}+r z \frac{\partial^{r-1}}{\partial y^{r-1}}\right) H^{\prime}\left(H^{-1}\left(\partial_{y}\right)\right)+\frac{A^{\prime}\left(H^{-1}\left(\partial_{y}\right)\right)}{A\left(H^{-1}\left(\partial_{y}\right)\right)}\right) H^{-1}\left(\partial_{y}\right)-n\right) \\
{ }_{L} H^{(m, r)} s_{n}(x, y, z)=0 .
\end{array}
$$


Proof. Using equations (2.8a) and (2.9a) in equation (1.10), we get assertion (2.18a) and similarly using equations $(2.8 \mathrm{~b})$ and $(2.9 \mathrm{~b})$ in equation $(1.10)$, we get assertion $(2.18 \mathrm{~b})$.

Remark 2.2. Since the Sheffer sequence $s_{n}(x)$ for $g(t)=1$ becomes the associated Sheffer sequence $\mathfrak{s}_{n}(x)$ for $f(t)$. (For our convenience, we denote the associated Sheffer sequence by $\mathfrak{s}_{n}(x)$ ). Therefore, for $g(t)=1$, we deduce the following consequences of Theorems 2.1-2.3:

Corollary 2.2. The Laguerre-Gould Hopper based associated Sheffer polynomials (LGHASP) ${ }_{L} H^{(m, r)} \mathfrak{s}_{n}(x, y, z)$ are defined by the generating function

$$
C_{0}\left(-x\left(f^{-1}(t)\right)^{m}\right) \exp \left(y f^{-1}(t)+z\left(f^{-1}(t)\right)^{r}\right)=\sum_{n=0}^{\infty}{ }_{L} H^{(m, r)} \mathfrak{s}_{n}(x, y, z) \frac{t^{n}}{n !},
$$

or, equivalently

$$
C_{0}\left(-x(H(t))^{m}\right) \exp \left(y H(t)+z(H(t))^{r}\right)=\sum_{n=0}^{\infty}{ }_{L} H^{(m, r)} \mathfrak{s}_{n}(x, y, z) \frac{t^{n}}{n !} .
$$

Corollary 2.3. The Laguerre-Gould Hopper based associated Sheffer polynomials ${ }_{L} H^{(m, r)} \mathfrak{s}_{n}(x, y, z)$ are quasi-monomial under the action of the following multiplicative and derivative operators:

$$
\hat{M}_{L H \mathfrak{s}}=\left(y+m D_{x}^{-1} \frac{\partial^{m-1}}{\partial y^{m-1}}+r z \frac{\partial^{r-1}}{\partial y^{r-1}}\right) \frac{1}{f^{\prime}\left(\partial_{y}\right)}
$$

or, equivalently

$$
\hat{M}_{L H \mathfrak{s}}=\left(y+m D_{x}^{-1} \frac{\partial^{m-1}}{\partial y^{m-1}}+r z \frac{\partial^{r-1}}{\partial y^{r-1}}\right) H^{\prime}\left(H^{-1}\left(\partial_{y}\right)\right)
$$

and

$$
\hat{P}_{L H \mathfrak{s}}=f\left(\partial_{y}\right)
$$

or, equivalently

$$
\hat{P}_{L H \mathfrak{s}}=H^{-1}\left(\partial_{y}\right)
$$

respectively.

Corollary 2.4. The Laguerre-Gould Hopper based associated Sheffer polynomials ${ }_{L} H^{(m, r)} \mathfrak{s}_{n}(x, y, z)$ satisfy the following differential equation:

$$
\left(\left(y+m D_{x}^{-1} \frac{\partial^{m-1}}{\partial y^{m-1}}+r z \frac{\partial^{r-1}}{\partial y^{r-1}}\right) \frac{f\left(\partial_{y}\right)}{f^{\prime}\left(\partial_{y}\right)}-n\right)_{L} H^{(m, r)} \mathfrak{s}_{n}(x, y, z)=0
$$


or, equivalently

$$
\left(\left(\left(y+m D_{x}^{-1} \frac{\partial^{m-1}}{\partial y^{m-1}}+r z \frac{\partial^{r-1}}{\partial y^{r-1}}\right) H^{\prime}\left(H^{-1}\left(\partial_{y}\right)\right)\right) H^{-1}\left(\partial_{y}\right)-n\right)_{L} H^{(m, r)} \mathfrak{s}_{n}(x, y, z)=0 .
$$

Remark 2.3. Since, for $f(t)=t$, the Sheffer polynomials $s_{n}(x)$ reduce to the Appell polynomials $A_{n}(x)$ [2]. Therefore, taking $f(t)=t$ in Theorems 2.1-2.3, we obtain the corresponding results for the Laguerre-Gould Hopper based Appell polynomials (LGHAP) ${ }_{L} H^{(m, r)} A_{n}(x, y, z)$ [34].

In the next section, we consider certain new and known families of special polynomials related to the Sheffer sequences and obtain the results for these mixed type special polynomials.

\section{Examples}

In Table 1, we have mentioned special cases of the $\operatorname{LGHP}{ }_{L} H_{n}^{(m, r)}(x, y, z)$. In order to obtain the results for the corresponding new or known special polynomials related to the Sheffer sequences, we consider the following examples:

Example 1. Since, for $m=1, r=2, x \rightarrow-x$, the LGHP ${ }_{L} H_{n}^{(m, r)}(x, y, z)$ reduce to the 3-variable Laguerre-Hermite polynomials (3VLHP) ${ }_{L} H_{n}(x, y, z)$ (Table 1(I)). Therefore, for the same choice of $m, r$ and $x$, the LGHSP ${ }_{L} H^{(m, r)} s_{n}(x, y, z)$ reduce to the 3 -variable Laguerre-Hermite based Sheffer polynomials (3VLHSP) ${ }_{L} H s_{n}(x, y, z)$. Thus, by using these substitutions in Theorems 2.1, 2.2 and 2.3 , we get the following results for the $3 \operatorname{VLHSP}_{L} s_{n}(x, y, z)$ :

Table 2. Results for the VLLHSP $_{L}{ }_{H} s_{n}(x, y, z)$

\begin{tabular}{|c|c|c|}
\hline $\begin{array}{l}\text { S. } \\
\text { No. }\end{array}$ & Results & Mathematical Expressions \\
\hline \multirow[t]{2}{*}{1.} & Generating & $\frac{1}{g\left(f^{-1}(t)\right)} C_{0}\left(x\left(f^{-1}(t)\right)\right) \exp \left(y f^{-1}(t)+z\left(f^{-1}(t)\right)^{2}\right)$ \\
\hline & function & $=A(t) C_{0}(x(H(t))) \exp \left(y H(t)+z(H(t))^{2}\right)=\sum_{n=0}^{\infty}{ }_{L} H^{s} s_{n}(x, y, z) \frac{t^{n}}{n !}$ \\
\hline \multirow[t]{2}{*}{2.} & Multiplicative and & $\hat{M}=\left(y-D_{x}^{-1}+2 z \frac{\partial}{\partial y}-\frac{g^{\prime}(\partial y)}{g(\partial y)}\right) \frac{1}{f^{\prime}(\partial y)}$ \\
\hline & derivative operators & $=\left(y-D_{x}^{-1}+2 z \frac{\partial}{\partial y}\right) H^{\prime}\left(H^{-1}\left(\partial_{y}\right)\right)+\frac{A^{\prime}\left(H^{-1}\left(\partial_{y}\right)\right)}{A\left(H^{-1}\left(\partial_{y}\right)\right)}$, \\
\hline \multirow[t]{2}{*}{3.} & Differential & $\left(\left(y-D_{x}^{-1}+2 z \frac{\partial}{\partial y}-\frac{g^{\prime}(\partial y)}{g(\partial y)}\right) \frac{f\left(\partial_{y}\right)}{f^{\prime}(\partial y)}-n\right)_{L} H^{s_{n}}(x, y, z)=0$, or equivalently \\
\hline & equation & $\left(\left(\left(y-D_{x}^{-1}+2 z \frac{\partial}{\partial y}\right) H^{\prime}\left(H^{-1}\left(\partial_{y}\right)\right)+\frac{A^{\prime}\left(H^{-1}\left(\partial_{y}\right)\right)}{A\left(H^{-1}\left(\partial_{y}\right)\right)}\right) H^{-1}\left(\partial_{y}\right)-n\right){ }_{L} H^{s_{n}}(x, y, z)=0$ \\
\hline 4. & Explicit & ${ }_{L} H^{s_{n}}(x, y, z)=\left(\left(y-D_{x}^{-1}+2 z \frac{\partial}{\partial y}-\frac{g^{\prime}(\partial y)}{g(\partial y)}\right) \frac{1}{f^{\prime}(\partial y)}\right)^{n}\{1\}$ \\
\hline & representation & $=\left(\left(y-D_{x}^{-1}+2 z \frac{\partial}{\partial y}\right) H^{\prime}\left(H^{-1}\left(\partial_{y}\right)\right)+\frac{A^{\prime}\left(H^{-1}\left(\partial_{y}\right)\right)}{A\left(H^{-1}\left(\partial_{y}\right)\right)}\right)^{n}\{1\}$ \\
\hline
\end{tabular}

Example 2. Since, for $m=1, r=2, x \rightarrow-x, z=-\frac{1}{2}$, the $\operatorname{LGHP}_{L} H_{n}^{(m, r)}(x, y, z)$ reduce to the 2-variable Laguerre-Hermite polynomials $(2 \mathrm{VLHP}){ }_{L} H_{n}^{\star}(x, y)$ (Table $1(\mathrm{II})$ ). Therefore, for the same choice of $m, r, x$ and $z$, the LGHSP ${ }_{L} H^{(m, r)} s_{n}(x, y, z)$ reduce to the 2-variable Laguerre-Hermite based Sheffer polynomials (2VLHSP) ${ }_{L} H^{\star} s_{n}(x, y)$. Thus, by using these substitutions in Theorems 
2.1, 2.2 and 2.3, we get the following results for the $2 \operatorname{VLHSP}_{L} H^{\star} s_{n}(x, y)$ :

Table 3. Results for the $2 \operatorname{VLHSP}_{L H^{\star}} s_{n}(x, y)$

\begin{tabular}{|c|c|c|}
\hline $\begin{array}{l}\text { S. } \\
\text { No. }\end{array}$ & Results & Mathematical Expressions \\
\hline \multirow[t]{2}{*}{1.} & Generating & $\frac{1}{g\left(f^{-1}(t)\right)} C_{0}\left(x\left(f^{-1}(t)\right)\right) \exp \left(y f^{-1}(t)-\frac{1}{2}\left(f^{-1}(t)\right)^{2}\right)$ \\
\hline & function & $=A(t) C_{0}(x(H(t))) \exp \left(y H(t)-\frac{1}{2}(H(t))^{2}\right)=\sum_{n=0}^{\infty}{ }_{L} H^{\star} s_{n}(x, y) \frac{t^{n}}{n !}$ \\
\hline \multirow[t]{2}{*}{2.} & Multiplicative and & $\hat{M}=\left(y-D_{x}^{-1}-\frac{\partial}{\partial y}-\frac{g^{\prime}\left(\partial_{y}\right)}{g(\partial y)}\right) \frac{1}{f^{\prime}(\partial y)}$ \\
\hline & derivative operators & $=\left(y-D_{x}^{-1}-\frac{\partial}{\partial y}\right) H^{\prime}\left(H^{-1}\left(\partial_{y}\right)\right)+\frac{A^{\prime}\left(H^{-1}\left(\partial_{y}\right)\right)}{A\left(H^{-1}\left(\partial_{y}\right)\right)}$ \\
\hline \multirow[t]{2}{*}{3.} & Differential & $\left(\left(y-D_{x}^{-1}-\frac{\partial}{\partial y}-\frac{g^{\prime}(\partial y)}{g(\partial y)}\right) \frac{f(\partial y)}{f^{\prime}(\partial y)}-n\right)_{L} H^{\star} s_{n}(x, y)=0$, or equivalently \\
\hline & equation & $\left(\left(\left(y-D_{x}^{-1}-\frac{\partial}{\partial y}\right) H^{\prime}\left(H^{-1}(\partial y)\right)+\frac{A^{\prime}\left(H^{-1}\left(\partial_{y}\right)\right)}{A\left(H^{-1}\left(\partial_{y}\right)\right)}\right) H^{-1}\left(\partial_{y}\right)-n\right){ }_{L} H^{\star} s_{n}(x, y)=0$ \\
\hline \multirow[t]{2}{*}{4.} & Explicit & $L H^{\star} s_{n}(x, y)=\left(\left(y-D_{x}^{-1}-\frac{\partial}{\partial y}-\frac{g^{\prime}\left(\partial_{y}\right)}{g(\partial y)}\right) \frac{1}{f^{\prime}(\partial y)}\right)^{n}\{1\}$ \\
\hline & representation & $=\left(\left(y-D_{x}^{-1}-\frac{\partial}{\partial y}\right) H^{\prime}\left(H^{-1}\left(\partial_{y}\right)\right)+\frac{A^{\prime}\left(H^{-1}\left(\partial_{y}\right)\right)}{A\left(H^{-1}\left(\partial_{y}\right)\right)}\right)^{n}\{1\}$ \\
\hline
\end{tabular}

Example 3. Since, for $m=1, r=2, x \rightarrow-x, y=1, z \rightarrow y$, the $\operatorname{LGHP}{ }_{L} H_{n}^{(m, r)}(x, y, z)$ reduce to the Laguerre-Hermite type polynomials (LHTP) $\varphi_{n}(x, y)$ (Table 1(III)). Therefore, for the same choice of $m, r, x, y$ and $z$, the $\operatorname{LGHSP}_{L} H^{(m . r)} s_{n}(x, y, z)$ reduce to the 2-variable Laguerre-Hermite type based Sheffer polynomials (LHTSP) $s_{n}(x, y)$. Thus, by using these substitutions in Theorems $2.1,2.2$ and 2.3 , we get the following results for the $2 \operatorname{VLHTSP}_{\varphi} s_{n}(x, y)$ :

Table 4. Results for the 2VLHTSP ${ }_{\varphi} s_{n}(x, y)$

\begin{tabular}{|c|c|c|}
\hline $\begin{array}{l}\text { S. } \\
\text { No. }\end{array}$ & Results & Mathematical Expressions \\
\hline \multirow[t]{2}{*}{1.} & Generating & $\frac{1}{g\left(f^{-1}(t)\right)} C_{0}\left(x\left(f^{-1}(t)\right)\right) \exp \left(f^{-1}(t)+y\left(f^{-1}(t)\right)^{2}\right)$ \\
\hline & function & $=A(t) C_{0}(x(H(t))) \exp \left(H(t)+y(H(t))^{2}\right)=\sum_{n=0}^{\infty} \varphi^{s_{n}}(x, y) \frac{t^{n}}{n !}$ \\
\hline \multirow[t]{3}{*}{2.} & Multiplicative and & $\hat{M}=\left(1-D_{x}^{-1}-2 y \frac{\partial}{\partial x} x \frac{\partial}{\partial x}-\frac{g^{\prime}\left(-\partial_{x} x \partial_{x}\right)}{g\left(-\partial_{x} x \partial_{x}\right)}\right) \frac{1}{f^{\prime}\left(-\partial_{x} x \partial_{x}\right)}$ \\
\hline & derivative operators & $=\left(1-D_{x}^{-1}-2 y \frac{\partial}{\partial x} x \frac{\partial}{\partial x}\right) H^{\prime}\left(H^{-1}\left(-\partial_{x} x \partial_{x}\right)\right)+\frac{A^{\prime}\left(H^{-1}\left(-\partial_{x} x \partial_{x}\right)\right)}{A\left(H^{-1}\left(-\partial_{x} \partial_{x}\right)\right)}$, \\
\hline & & $\hat{P}=f\left(-\partial_{x} x \partial_{x}\right)=H^{-1}\left(-\partial_{x} x \partial_{x}\right)$ \\
\hline \multirow[t]{2}{*}{3.} & Differential & $\left(\left(1-D_{x}^{-1}-2 y \frac{\partial}{\partial x} x \frac{\partial}{\partial x}-\frac{g^{\prime}\left(-\partial_{x} x \partial_{x}\right)}{g\left(-\partial_{x} x \partial x\right)}\right) \frac{f\left(-\partial_{x} x \partial_{x}\right)}{f^{\prime}\left(-\partial_{x} x \partial_{x}\right)}-n\right) \varphi s_{n}(x, y)=0$, or equivalently \\
\hline & equation & $\left(\left(\left(1-D_{x}^{-1}-2 y \frac{\partial}{\partial x} x \frac{\partial}{\partial x}\right) H^{\prime}\left(H^{-1}\left(-\partial_{x} x \partial_{x}\right)\right)+\frac{A^{\prime}\left(H^{-1}\left(-\partial_{x} x \partial_{x}\right)\right)}{A\left(H^{-1}\left(-\partial_{x} x \partial_{x}\right)\right)}\right) H^{-1}\left(-\partial_{x} x \partial_{x}\right)-n\right)$ \\
\hline 4. & Explicit & $\varphi s_{n}(x, y)=\left(\left(1-D_{x}^{-1}-2 y \frac{\partial}{\partial x} x \frac{\partial}{\partial x}-\frac{g^{\prime}\left(-\partial_{x} x \partial x\right)}{g\left(-\partial_{x} x \partial x\right)}\right) \frac{1}{f^{\prime}\left(-\partial_{x} x \partial_{x}\right)}\right)^{n}\{1\}$ \\
\hline & representation & $=\left(\left(1-D_{x}^{-1}-2 y \frac{\partial}{\partial x} x \frac{\partial}{\partial x}\right) H^{\prime}\left(H^{-1}\left(-\partial_{x} x \partial_{x}\right)\right)+\frac{A^{\prime}\left(H^{-1}\left(-\partial_{x} x \partial_{x}\right)\right)}{A\left(H^{-1}\left(-\partial_{x} x \partial x\right)\right)}\right)^{n}\{1\}$ \\
\hline
\end{tabular}

Example 4. Since, for $x=0$, the $\operatorname{LGHP}_{L} H_{n}^{(m, r)}(x, y, z)$ reduce to the Gould Hopper polynomials (GHP) $H_{n}^{(r)}(y, z)$ (Table 1(IV)). Therefore, for the same choice of $x$, the LGHSP ${ }_{L} H^{(m, r)} s_{n}(x, y, z)$ reduce to the Gould Hopper based Sheffer polynomials (GHSP) ${ }_{H^{(r)}} s_{n}(y, z)$ [35]. Thus, by using these substitutions in Theorems 2.1, 2.2 and 2.3, we obtain the corresponding results for the GHSP $H^{(r)} s_{n}(y, z)[35]$.

Example 5. Since, for $z=0$, the $\operatorname{LGHP}_{L} H_{n}^{(m, r)}(x, y, z)$ reduce to the 2-variable generalized 
Laguerre polynomials (2VLP) ${ }_{m} L_{n}(x, y)$ (Table $1(\mathrm{~V})$ ). Therefore, for the same choice of $z$, the LGHSP $_{L} H^{(m, r)} s_{n}(x, y, z)$ reduce to the 2-variable generalized Laguerre based Sheffer polynomials $(2 \mathrm{VGLSP})_{m} s_{n}(x, y)$. Thus, by using these substitutions in Theorems 2.1, 2.2 and 2.3 , we get the following results for the $2 \operatorname{VGLSP}_{m} L s_{n}(x, y)$ :

Table 5. Results for the 2VGLSP ${ }_{m} L s_{n}(x, y)$

\begin{tabular}{|c|c|c|}
\hline No. & Results & Mathematical Expressions \\
\hline \multirow[t]{2}{*}{1.} & Generating & $\frac{1}{g\left(f^{-1}(t)\right)} C_{0}\left(-x\left(f^{-1}(t)\right)^{m}\right) \exp \left(y f^{-1}(t)\right)$ \\
\hline & function & $=A(t) C_{0}\left(-x(H(t))^{m}\right) \exp (y H(t))=\sum_{n=0}^{\infty} m L^{s_{n}}(x, y) \frac{t^{n}}{n !}$ \\
\hline \multirow[t]{2}{*}{2.} & Multiplicative and & $\hat{M}=\left(y+m D_{x}^{-1} \frac{\partial^{m-1}}{\partial y^{m-1}}-\frac{g^{\prime}\left(\partial_{y}\right)}{g\left(\partial_{y}\right)}\right) \frac{1}{f^{\prime}\left(\partial_{y}\right)}$ \\
\hline & derivative operators & $=\left(y+m D_{x}^{-1} \frac{\partial^{m-1}}{\partial y^{m-1}}\right) H^{\prime}\left(H^{-1}\left(\partial_{y}\right)\right)+\frac{A^{\prime}\left(H^{-1}\left(\partial_{y}\right)\right)}{A\left(H^{-1}\left(\partial_{y}\right)\right)}$ \\
\hline \multirow[t]{2}{*}{3.} & Differential & $\left(\left(y+m D_{x}^{-1} \frac{\partial^{m-1}}{\partial y m-1}-\frac{g^{\prime}\left(\partial_{y}\right)}{g(\partial y)}\right) \frac{f\left(\partial_{y}\right)}{f^{\prime}(\partial y)}-n\right){ }_{m} L^{s_{n}}(x, y)=0$, or equivalently \\
\hline & equation & $\left(\left(\left(y+m D_{x}^{-1} \frac{\partial^{m-1}}{\partial y^{m-1}}\right) H^{\prime}\left(H^{-1}\left(\partial_{y}\right)\right)+\frac{A^{\prime}\left(H^{-1}\left(\partial_{y}\right)\right)}{A\left(H^{-1}\left(\partial_{y}\right)\right)}\right) H^{-1}\left(\partial_{y}\right)-n\right){ }_{m} L^{s_{n}}(x, y)=0$ \\
\hline \multirow[t]{2}{*}{4.} & Explicit & $m L^{s_{n}(x, y)}=\left(\left(y+m D_{x}^{-1} \frac{\partial^{m-1}}{\partial y^{m-1}}-\frac{g^{\prime}(\partial y)}{g(\partial y)}\right) \frac{1}{f^{\prime}(\partial y)}\right)^{n}\{1\}$ \\
\hline & representation & $=\left(\left(y+m D_{x}^{-1} \frac{\partial^{m-1}}{\partial y^{m-1}}\right) H^{\prime}\left(H^{-1}\left(\partial_{y}\right)\right)+\frac{A^{\prime}\left(H^{-1}\left(\partial_{y}\right)\right)}{A\left(H^{-1}\left(\partial_{y}\right)\right)}\right)^{n}\{1\}$ \\
\hline
\end{tabular}

Example 6. Since, for $r=m, x=0, y \rightarrow-D_{x}^{-1}, z \rightarrow y$, the $\operatorname{LGHP}_{L} H_{n}^{(m, r)}(x, y, z)$ reduce to the 2 -variable generalized Laguerre type polynomials $\left(2 \mathrm{VGLTP}{ }_{[m]} L_{n}(x, y)\right.$ (Table $1(\mathrm{VI})$ ). Therefore, for the same choice of $r, x, y$ and $z$, the $\operatorname{LGHSP}_{L} H^{(m, r)} s_{n}(x, y, z)$ reduce to the 2-variable generalized Laguerre type based Sheffer polynomials (2VGLTSP) ${ }_{[m]}{ }^{L} s_{n}(x, y)$. Thus, by using these substitutions in Theorems 2.1, 2.2 and 2.3, we get the following results for the $2 \operatorname{VGLTSP}_{[m]} L_{n}(x, y)$ :

Table 6. Results for the 2VGLTSP ${ }_{[m]}{ } s_{n}(x, y)$

\begin{tabular}{|c|c|c|}
\hline $\begin{array}{l}\text { S. } \\
\text { No. }\end{array}$ & Results & Mathematical Expressions \\
\hline \multirow[t]{2}{*}{1.} & Generating & $\frac{1}{g\left(f^{-1}(t)\right)} C_{0}\left(x f^{-1}(t)\right) \exp \left(y\left(f^{-1}(t)\right)^{m}\right)$ \\
\hline & function & 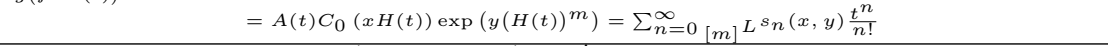 \\
\hline 2. & Multiplicative and & $\begin{aligned} \hat{M} & =\left(-D_{x}^{-1}+(-1)^{m} m y \frac{\partial^{m-1}}{\partial x^{m-1}} x^{m-1} \frac{\partial^{m-1}}{\partial x^{m-1}}-\frac{g^{\prime}\left(-\partial_{x} x \partial_{x}\right)}{g\left(-\partial_{x} x \partial_{x}\right)}\right) \frac{1}{f^{\prime}\left(-\partial_{x} x \partial_{x}\right)} \\
& =\left(-D_{x}^{-1}+(-1)^{m} m y \frac{\partial^{m-1}}{\partial x^{m-1}} x^{m-1} \frac{\partial^{m-1}}{\partial x^{m-1}}\right) H^{\prime}\left(H^{-1}\left(-\partial_{x} x \partial_{x}\right)\right)+\frac{A^{\prime}\left(H^{-1}\left(-\partial_{x} x \partial_{x}\right)\right)}{A\left(H^{-1}\left(-\partial_{x} x \partial_{x}\right)\right)}, \\
\hat{P} & =f\left(-\partial_{x} x \partial_{x}\right)=H^{-1}\left(-\partial_{x} x \partial_{x}\right)\end{aligned}$ \\
\hline 3. & $\begin{array}{l}\text { Differential } \\
\text { equation }\end{array}$ & $\begin{array}{c}\left(\left(-D_{x}^{-1}+(-1)^{m} m y \frac{\partial^{m-1}}{\partial x^{m-1}} x^{m-1} \frac{\partial^{m-1}}{\partial x^{m-1}}-\frac{g^{\prime}\left(-\partial_{x} x \partial_{x}\right)}{g\left(-\partial_{x} x \partial x\right.}\right) \frac{f\left(-\partial_{x} x \partial_{x}\right)}{f^{\prime}\left(-\partial_{x} x \partial_{x}\right)}-n\right){ }_{[m]} L^{s_{n}}(x, y)=0 \text {, or } \\
\left(\left(\left(y+m D_{x}^{-1} \frac{\partial^{m-1}}{\partial y^{m-1}}\right) H^{\prime}\left(H^{-1}\left(-\partial_{x} x \partial_{x}\right)\right)+\frac{A^{\prime}\left(H^{-1}\left(-\partial_{x} x \partial_{x}\right)\right)}{A\left(H^{-1}\left(-\partial_{x} x \partial_{x}\right)\right)}\right) H^{-1}\left(-\partial_{x} x \partial_{x}\right)-n\right) \\
{[m]^{s_{n}(x, y)=0}}\end{array}$ \\
\hline 4. & representation & $\begin{array}{l}{[m] L^{s_{n}(x, y)}=\left(\left(-D_{x}^{-1}+(-1)^{m} m y \frac{\partial^{m-1}}{\partial x^{m-1} x^{m-1}} \frac{\partial^{m-1}}{\partial x^{m-1}}-\frac{g^{\prime}\left(-\partial_{x} x \partial_{x}\right)}{g\left(-\partial_{x} x \partial_{x}\right)}\right) \frac{1}{f^{\prime}\left(-\partial_{x} x \partial_{x}\right)}\right)^{n}\{1\}} \\
=\left(\left(-D_{x}^{-1}+(-1)^{m} m y \frac{\partial^{m-1}}{\partial x^{m-1}} x^{m-1} \frac{\partial^{m-1}}{\partial x^{m-1}}\right) H^{\prime}\left(H^{-1}\left(-\partial_{x} x \partial_{x}\right)\right)+\frac{A^{\prime}\left(H^{-1}\left(-\partial_{x} x \partial_{x}\right)\right)}{A\left(H^{-1}\left(-\partial_{x} x \partial_{x}\right)\right)}\right)^{n}\{1\}\end{array}$ \\
\hline
\end{tabular}

Example 7. Since, for $r=m-1, x=0, y \rightarrow x, z \rightarrow y$, the $\operatorname{LGHP}_{L} H_{n}^{(m, r)}(x, y, z)$ reduce to the generalized Chebyshev polynomials $(\mathrm{GCP}) U_{n}^{(m)}(x, y)$ (Table $1(\mathrm{VII})$ ). Therefore, for the same choice of $r, x, y$ and $z$, the LGHSP ${ }_{L} H^{(m, r)} s_{n}(x, y, z)$ reduce to the generalized Chebyshev based Sheffer polynomials $(\mathrm{GCSP})_{U^{(m)}} s_{n}(x, y)[30]$. Thus, by using these substitutions in Theorems 2.1, 
2.2 and 2.3, we obtain the corresponding results for the $\operatorname{GCSP}_{U^{(m)}} s_{n}(x, y)[30]$.

Example 8. Since, for $m=1, x \rightarrow-x, z=0$, the $\operatorname{LGHP}_{L} H_{n}^{(m, r)}(x, y, z)$ reduce to the 2-variable Laguerre polynomials (2VLP) $L_{n}(x, y)$ (Table 1(VIII)). Therefore, for the same choice of $m, x$ and $z$, the LGHSP ${ }_{L} H^{(m, r)} s_{n}(x, y, z)$ reduce to the 2-variable Laguerre based Sheffer polynomials $(2 \mathrm{VLSP})_{L} s_{n}(x, y)$ [32]. Thus, by using these substitutions in Theorems 2.1, 2.2 and 2.3, we obtain the corresponding results for the $2 \operatorname{VLSP}{ }_{L} s_{n}(x, y)[32]$.

Example 9. Since, for $m=1, x \rightarrow y, y \rightarrow-D_{x}^{-1}, z=0$, the $\operatorname{LGHP}_{L} H_{n}^{(m, r)}(x, y, z)$ reduce to the 2-variable Legendre polynomials (2VLeP) $\frac{R_{n}(x, y)}{n !}$ (Table $1(\mathrm{IX})$ ). Therefore, for the same choice of $m, x, y$ and $z$, the LGHSP ${ }_{L} H^{(m, r)} s_{n}(x, y, z)$ reduce to the 2-variable Legendre based Sheffer polynomials (2VLeSP) $\frac{R s_{n}(x, y)}{n !}$ [33]. Thus, by using these substitutions in Theorems 2.1, 2.2 and 2.3 , we obtain the corresponding results for the 2VLeSP $\frac{R s_{n}(x, y)}{n !}$ [33].

Example 10. Since, for $x=0, y \rightarrow x, z \rightarrow y \partial_{y} y$, the $\operatorname{LGHP}{ }_{L} H_{n}^{(m, r)}(x, y, z)$ reduce to the 2 -variable truncated polynomials of order $r(2 \mathrm{VTP}) e_{n}^{(r)}(x, y)$ (Table $1(\mathrm{X})$ ). Therefore, for the same choice of $x, y$ and $z$, the LGHSP ${ }_{L} H^{(m, r)} s_{n}(x, y, z)$ reduce to the 2-variable truncated exponential based Sheffer polynomials (2VTESP) $e^{(r)} s_{n}(x, y)$ [36]. Thus, by using these substitutions in Theorems 2.1, 2.2 and 2.3, we obtain the corresponding results for the $2 \operatorname{VTESP}_{e^{(r)}} s_{n}(x, y)[36]$.

Example 11. Since, for $r=2, x=0$, the $\operatorname{LGHP}_{L} H_{n}^{(m, r)}(x, y, z)$ reduce to the 2 -variable Hermite Kampé de Fériet polynomials $(2 \mathrm{VHKdFP}) H_{n}(y, z)$ (Table $1(\mathrm{XI})$ ). Therefore, for the same choice of $r$ and $x$, the LGHSP ${ }_{L} H^{(m, r)} s_{n}(x, y, z)$ reduce to the 2-variable Hermite Kampé de Fériet based Sheffer polynomials (2VHKdFSP) ${ }_{H} s_{n}(y, z)$ [31]. Thus, by using these substitutions in Theorems 2.1, 2.2 and 2.3, we obtain the corresponding results for the $2 \operatorname{VHKdFSP}_{H} s_{n}(y, z)[31]$.

Example 12. Since, for $r=2, x=0, y \rightarrow D_{x}^{-1}, z \rightarrow y$, the $\operatorname{LGHP}{ }_{L} H_{n}^{(m, r)}(x, y, z)$ reduce to the Hermite type polynomials (HTP) $G_{n}(x, y)$ (Table $1(\mathrm{XII})$ ). Therefore, for the same choice of $r, x, y$ and $z$, the LGHSP ${ }_{L} H^{(m, r)} s_{n}(x, y, z)$ reduce to the Hermite type based Sheffer polynomials (HTSP) ${ }_{G} s_{n}(x, y)$. Thus, by using these substitutions in Theorems 2.1, 2.2 and 2.3, we get the following results for the $\operatorname{HTSP}_{G} s_{n}(x, y)$ : 
Table 7. Results for the $\operatorname{HTSP}_{G} s_{n}(x, y)$

\begin{tabular}{|c|c|c|}
\hline $\begin{array}{l}\text { S. } \\
\text { No. }\end{array}$ & Results & Mathematical Expressions \\
\hline \multirow[t]{2}{*}{1.} & Generating & $\frac{1}{g\left(f^{-1}(t)\right)} C_{0}\left(-x f^{-1}(t)\right) \exp \left(y\left(f^{-1}(t)\right)^{2}\right)$ \\
\hline & function & $=A(t) C_{0}(-x H(t)) \exp \left(y(H(t))^{2}\right)=\sum_{n=0}^{\infty} G^{s} n(x, y) \frac{t^{n}}{n !}$ \\
\hline \multirow[t]{3}{*}{2.} & Multiplicative and & $\hat{M}=\left(D_{x}^{-1}+2 y \frac{\partial}{\partial x} x \frac{\partial}{\partial x}-\frac{g^{\prime}\left(\partial_{x} x \partial_{x}\right)}{g\left(\partial_{x} x \partial x\right)}\right) \frac{1}{f^{\prime}\left(\partial_{x} x \partial_{x}\right)}$ \\
\hline & & $=\left(D_{x}^{-1}+2 y \frac{\partial}{\partial x} x \frac{\partial}{\partial x}\right) H^{\prime}\left(H^{-1}\left(\partial_{x} x \partial_{x}\right)\right)+\frac{A^{\prime}\left(H^{-1}\left(\partial_{x} x \partial_{x}\right)\right)}{A\left(H^{-1}\left(\partial_{x} x \partial_{x}\right)\right)}$, \\
\hline & derivative operators & $\hat{P}=f\left(\partial_{x} x \partial_{x}\right)=H^{-1}\left(\partial_{x} x \partial_{x}\right)$ \\
\hline \multirow[t]{2}{*}{3.} & Differential & $\left(\left(D_{x}^{-1}+2 y \frac{\partial}{\partial x} x \frac{\partial}{\partial x}-\frac{g^{\prime}\left(\partial_{x} x \partial_{x}\right)}{g\left(\partial_{x} x \partial x\right)}\right) \frac{f\left(\partial_{x} x \partial_{x}\right)}{f^{\prime}\left(\partial_{x} x \partial_{x}\right)}-n\right){ }_{G} s_{n}(x, y)=0$, or equivalently \\
\hline & equation & $\left(\left(\left(D_{x}^{-1}+2 y \frac{\partial}{\partial x} x \frac{\partial}{\partial x}\right) H^{\prime}\left(H^{-1}\left(\partial_{x} x \partial_{x}\right)\right)+\frac{A^{\prime}\left(H^{-1}\left(\partial_{x} x \partial_{x}\right)\right)}{A\left(H^{-1}\left(\partial_{x} x \partial_{x}\right)\right)}\right) H^{-1}\left(\partial_{x} x \partial_{x}\right)-n\right)$ \\
\hline 4 & & $\begin{array}{lll} & & G_{n}(x, y)=0\end{array}$ \\
\hline 4. & $\begin{array}{l}\text { Explicit } \\
\text { representation }\end{array}$ & 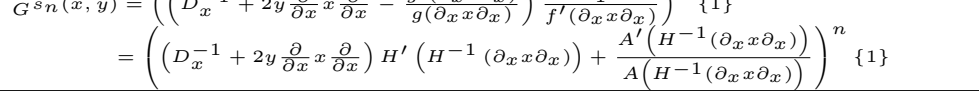 \\
\hline
\end{tabular}

Example 13. Since, for $m=2, x \rightarrow\left(\frac{x^{2}-1}{4}\right), y \rightarrow x, z=0$, the $\operatorname{LGHP}_{L} H_{n}^{(m, r)}(x, y, z)$ reduce to the Legendre polynomials (LeP) $P_{n}(x)$ (Table $1(\mathrm{XIII})$ ). Therefore for the same substitutions of $m$, $x, y$ and $z$, the LGHSP ${ }_{L} H^{(m, r)} s_{n}(x, y, z)$ reduce to the Legendre based Sheffer polynomials (LeSP) ${ }_{P} s_{n}(x)$. Thus, by using these substitutions in Theorems 2.1, 2.2 and 2.3, we get the following results for the $\operatorname{LeSP}_{P} s_{n}(x)$ :

Table 8. Results for the $\operatorname{LeSP}_{P} s_{n}(x)$

\begin{tabular}{|c|c|c|}
\hline $\begin{array}{l}\text { S. } \\
\text { No. }\end{array}$ & Results & Mathematical Expressions \\
\hline 1. & $\begin{array}{l}\text { Generating } \\
\text { function }\end{array}$ & $\begin{aligned} \frac{1}{g\left(f^{-1}(t)\right)} C_{0}\left(-\left(\frac{x^{2}-1}{4}\right)\left(f^{-1}(t)\right)^{2}\right) \exp \left(x f^{-1}(t)\right) \\
\\
=A(t) C_{0}\left(-\left(\frac{x^{2}-1}{4}\right)(H(t))^{2}\right) \exp (x H(t))=\sum_{n=0}^{\infty} P^{s} n(x) \frac{t^{n}}{n !}\end{aligned}$ \\
\hline 2. & derivative operators & $\begin{aligned} & \hat{M}=(x+2 D-1 \\
&\left.\left(\frac{x^{2}-1}{4}\right)^{\frac{\partial}{\partial x}}-\frac{g^{\prime}\left(\partial_{x}\right)}{g(\partial x)}\right) \frac{1}{f^{\prime}\left(\partial_{x}\right)} \\
&=\left(x+2 D^{-1}\left(\frac{x^{2}-1}{4}\right)^{\frac{\partial}{\partial x}}\right) H^{\prime}\left(H^{-1}\left(\partial_{x}\right)\right)+\frac{A^{\prime}\left(H^{-1}\left(\partial_{x}\right)\right)}{A\left(H^{-1}\left(\partial_{x}\right)\right)}, \\
& \hat{P}=f\left(\partial_{x}\right)=H^{-1}\left(\partial_{x}\right)\end{aligned}$ \\
\hline 3. & Differential & $\begin{array}{l}\left.\left(x+2 D^{-1}\left(\frac{x^{2}-1}{4}\right) \frac{\partial}{\partial x}-\frac{g^{\prime}\left(\partial_{x}\right)}{g\left(\partial_{x}\right)}\right) \frac{f\left(\partial_{x}\right)}{f^{\prime}\left(\partial_{x}\right)}-n\right) P^{s_{n}(x)=0, \text { or equivalently }} \\
\left.\left(\left(x+2 D^{-1}\left(\frac{x^{2}-1}{4}\right)^{\frac{\partial}{\partial x}}\right) H^{\prime}\left(H^{-1}\left(\partial_{x}\right)\right)+\frac{A^{\prime}\left(H^{-1}\left(\partial_{x}\right)\right)}{A\left(H^{-1}\left(\partial_{x}\right)\right)}\right) H^{-1}\left(\partial_{x}\right)-n\right) P^{s_{n}(x)=0}\end{array}$ \\
\hline 4. & representation & $\begin{aligned} P s_{n}(x) & =\left(\left(x+2 D^{-1}\left(\frac{x^{2}-1}{4}\right) \frac{\partial}{\partial x}-\frac{g^{\prime}\left(\partial_{x}\right)}{g(\partial x)}\right) \frac{1}{f^{\prime}\left(\partial_{x}\right)}\right)\{1\} \\
& =\left(\left(x+2 D^{-1}\left(\frac{x^{2}-1}{4}\right)^{\frac{\partial}{\partial x}}\right) H^{\prime}\left(H^{-1}\left(\partial_{x}\right)\right)+\frac{A^{\prime}\left(H^{-1}\left(\partial_{x}\right)\right)}{A\left(H^{-1}\left(\partial_{x}\right)\right)}\right)^{n}\{1\}\end{aligned}$ \\
\hline
\end{tabular}

Example 14. Since, for $x \rightarrow y \partial_{y} y, y \rightarrow x$, the $\operatorname{LGHP}_{L} H_{n}^{(m, r)}(x, y, z)$ reduce to the 3 -variable generalized Hermite polynomials (3VGHP) $H_{n}^{(r, m)}(x, y, z)$ (Table $1(\mathrm{XIV})$ ). Therefore, for the same choice of $x$ and $y$, the LGHSP ${ }_{L} H^{(m, r)} s_{n}(x, y, z)$ reduce to the 3 -variable generalized Hermite based 
Sheffer polynomials (3VGHSP) ${ }_{H^{(r, m)}} s_{n}(x, y, z)$. Thus, by using these substitutions in Theorems 2.1, 2.2 and 2.3, we get the following results for the $3 \operatorname{VGHSP}_{H^{(r, m)}} s_{n}(x, y, z)$ :

Table 9. Results for the 3 VGHSP $_{H^{(r, m)}} s_{n}(x, y, z)$

\begin{tabular}{|c|c|c|}
\hline No. & Results & Mathematical Expressions \\
\hline \multirow[t]{2}{*}{1.} & Generating & $\frac{1}{g\left(f^{-1}(t)\right)} \exp \left(x f^{-1}(t)+y\left(f^{-1}(t)\right)^{m}+z\left(f^{-1}(t)\right)^{r}\right)$ \\
\hline & function & $=A(t) \exp \left(x H(t)+y(H(t))^{m}+z(H(t))^{r}\right)=\sum_{n=0}^{\infty}{ }_{H}(r, m)^{s_{n}}(x, y, z) \frac{t^{n}}{n !}$ \\
\hline \multirow[t]{3}{*}{2.} & Multiplicative and & $\hat{M}=\left(x+m y \frac{\partial^{m-1}}{\partial x^{m-1}}+r z \frac{\partial^{r-1}}{\partial x^{r-1}}-\frac{g^{\prime}\left(\partial_{x}\right)}{g(\partial x)}\right) \frac{1}{f^{\prime}(\partial x)}$ \\
\hline & & $=\left(x+m y \frac{\partial^{m-1}}{\partial^{\prime}}+r z \frac{\partial^{r-1}}{\partial^{1}}\right) H^{\prime}\left(H^{-1}\left(\partial_{r}\right)\right)+\frac{A^{\prime}\left(H^{-1}\left(\partial_{x}\right)\right)}{}$ \\
\hline & derivative operators & $\hat{P}=f\left(\partial_{x}\right)=H^{-1}\left(\partial_{x}\right) \quad A\left(H^{-1}\left(\partial_{x}\right)\right)$ \\
\hline \multirow[t]{3}{*}{3.} & Differential & $\left(\left(x+m y \frac{\partial^{m-1}}{\partial x^{m-1}}+r z \frac{\partial^{r-1}}{\partial x^{r-1}}-\frac{g^{\prime}\left(\partial_{x}\right)}{g(\partial x)}\right) \frac{f\left(\partial_{x}\right)}{f^{\prime}\left(\partial_{x}\right)}-n\right)_{H}(r, m) s_{n}(x, y, z)=0$, or equivalently \\
\hline & equation & $\left(\left(\left(x+m y \frac{\partial^{m-1}}{\partial x^{m-1}}+r z \frac{\partial^{r-1}}{\partial x^{r-1}}\right) H^{\prime}\left(H^{-1}\left(\partial_{x}\right)\right)+\frac{A^{\prime}\left(H^{-1}\left(\partial_{x}\right)\right)}{A\left(H^{-1}\left(\partial_{x}\right)\right)}\right) H^{-1}\left(\partial_{x}\right)-n\right)$ \\
\hline & & $H^{(r, m)} s_{n}(x, y, z)=0$ \\
\hline 4. & representation & 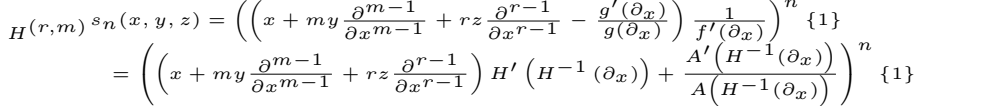 \\
\hline
\end{tabular}

Example 15. Since, for $m=2, r=3, x \rightarrow z \partial_{z} z, y \rightarrow x, z \rightarrow y$, the $\operatorname{LGHP}_{L} H_{n}^{(m, r)}(x, y, z)$ reduce to the Bell type polynomials (BTP) $H_{n}^{(3,2)}(x, y, z)$ (Table $\left.1(\mathrm{XV})\right)$. Therefore, for the same choice of $m, r, x, y$ and $z$, the LGHSP ${ }_{L} H^{(m, r)} s_{n}(x, y, z)$ reduce to the Bell type based Sheffer polynomials (BTSP) ${ }_{H^{(3,2)}} s_{n}(x, y, z)$. Thus, by using these substitutions in Theorems 2.1, 2.2 and 2.3, we get the following results for the $\operatorname{BTSP}_{H^{(3,2)}} s_{n}(x, y, z)$ :

Table 10. Results for the BTSP ${ }_{H^{(3,2)}} s_{n}(x, y, z)$

\begin{tabular}{|c|c|c|}
\hline $\begin{array}{l}\text { S. } \\
\text { No. }\end{array}$ & Results & Mathematical Expressions \\
\hline \multirow[t]{2}{*}{1.} & Generating & $\frac{1}{g\left(f^{-1}(t)\right)} \exp \left(x f^{-1}(t)+y\left(f^{-1}(t)\right)^{3}+z\left(f^{-1}(t)\right)^{2}\right)$ \\
\hline & function & $=A(t) \exp \left(x H(t)+y(H(t))^{3}+z(H(t))^{2}\right)=\sum_{n=0}^{\infty} H^{(3,2)} s_{n}(x, y, z) \frac{t^{n}}{n !}$ \\
\hline \multirow[t]{3}{*}{2.} & Multiplicative and & $\hat{M}=\left(x+3 y \frac{\partial^{2}}{\partial x^{2}}+2 z \frac{\partial}{\partial x}-\frac{g^{\prime}\left(\partial_{x}\right)}{g\left(\partial_{x}\right)}\right) \frac{1}{f^{\prime}\left(\partial_{x}\right)}$ \\
\hline & & 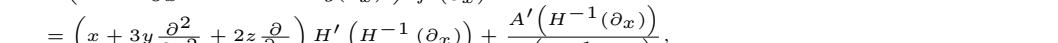 \\
\hline & derivative operators & $\hat{P}=f\left(\partial_{x}\right)=H^{-1}\left(\partial_{x}\right)$ \\
\hline \multirow[t]{2}{*}{3.} & Differential & $\left(\left(x+3 y \frac{\partial^{2}}{\partial x^{2}}+2 z \frac{\partial}{\partial x}-\frac{g^{\prime}\left(\partial_{x}\right)}{g\left(\partial_{x}\right)}\right) \frac{f\left(\partial_{x}\right)}{f^{\prime}\left(\partial_{x}\right)}-n\right)_{H}(3,2) s_{n}(x, y, z)=0$, or equivalently \\
\hline & equation & 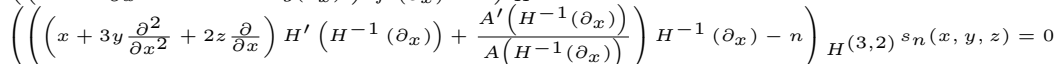 \\
\hline 4. & $\begin{array}{l}\text { Explicit } \\
\text { representation }\end{array}$ & $\begin{aligned} H^{(3,2)} s_{n}(x, y, z)=\left(\left(x+3 y \frac{\partial^{2}}{\partial x^{2}}+2 z \frac{\partial}{\partial x}-\frac{g^{\prime}\left(\partial_{x}\right)}{g(\partial x)}\right) \frac{1}{f^{\prime}\left(\partial_{x}\right)}\right)^{n}\{1\} \\
=\left(\left(x+3 y \frac{\partial^{2}}{\partial x^{2}}+2 z \frac{\partial}{\partial x}\right) H^{\prime}\left(H^{-1}\left(\partial_{x}\right)\right)+\frac{A^{\prime}\left(H^{-1}\left(\partial_{x}\right)\right)}{A\left(H^{-1}\left(\partial_{x}\right)\right)}\right)^{n}\{1\}\end{aligned}$ \\
\hline
\end{tabular}

Remark 3.1. For $\frac{1}{g\left(f^{-1}(t)\right)}=A(t)=1$, the above mentioned special cases (Examples 1-15) of the LGHSP $_{L} H^{(m, r)} s_{n}(x, y, z)$ yield the corresponding results for the LGHASP ${ }_{L} H^{(m, r)} \mathfrak{s}_{n}(x, y, z)$, which can also be deduced by using certain substitutions in Corollaries 2.2-2.4.

Remark 3.2. For $f^{-1}(t)=H(t)=t$, the above mentioned special cases (Examples 1-15) of the LGHSP $_{L} H^{(m, r)} s_{n}(x, y, z)$ yield the corresponding results for the LGHAP ${ }_{L} H^{(m, r)} A_{n}(x, y, z)[34]$. 
In the next section, we derive certain operational and integral representations for the LGHSP ${ }_{L} H^{(m, r)} s_{n}(x, y, z)$.

\section{Operational and integral representations}

To establish the operational representations for the $\operatorname{LGHSP}_{L} H^{(m, r)} s_{n}(x, y, z)$, we prove the following results:

Theorem 4.1. The following operational representation connecting the $L G H S P{ }_{L} H^{(m, r)} s_{n}(x, y, z)$ with the Sheffer polynomials $s_{n}(x)$ holds true:

$$
{ }_{L} H^{(m, r)} s_{n}(x, y, z)=\exp \left(D_{x}^{-1} \frac{\partial^{m}}{\partial y^{m}}+z \frac{\partial^{r}}{\partial y^{r}}\right) s_{n}(y)
$$

Proof. In view of equation (2.7), the proof is direct use of identity (2.4).

Theorem 4.2. The following operational representation connecting the $L G H S P{ }_{L} H^{(m, r)} s_{n}(x, y, z)$ with the $2 V G L S P_{m} L s_{n}(x, y)$ holds true:

$$
{ }_{L} H^{(m, r)} s_{n}(x, y, z)=\exp \left(z \frac{\partial^{r}}{\partial y^{r}}\right){ }_{m}{ }_{L} s_{n}(x, y)
$$

Proof. From equation (2.1) (or (2.2)), we have

$$
\frac{\partial^{r}}{\partial y^{r}}{ }_{L} H^{(m, r)} s_{n}(x, y, z)={\frac{\partial}{\partial z^{L}} H^{(m, r)}} s_{n}(x, y, z) .
$$

Since, in view of Table $1(\mathrm{~V})$, we have

$$
{ }_{L} H_{n}^{(m, r)}(x, y, 0)={ }_{m} L_{n}(x, y) .
$$

Therefore, from Example 5 of Section 3, we have

$$
{ }_{L} H^{(m, r)} s_{n}(x, y, 0)={ }_{m} L s_{n}(x, y) .
$$

Now, solving equations (4.3) subject to initial condition (4.5), we get assertion (4.2).

Theorem 4.3. The following operational representation connecting the $L G H S P{ }_{L} H^{(m, r)} s_{n}(x, y, z)$ with the $\operatorname{GHSP}_{H^{(r)}} s_{n}(y, z)$ holds true:

$$
{ }_{L} H^{(m, r)} s_{n}(x, y, z)=\exp \left(D_{x}^{-1} \frac{\partial^{m}}{\partial y^{m}}\right){ }_{H^{(r)}} s_{n}(y, z) .
$$

Proof. From equations (1.15) and (2.1) (or (2.2)), we have

$$
\frac{\partial^{m}}{\partial y^{m}}{ }_{L} H^{(m . r)} s_{n}(x, y, z)=\frac{\partial}{\partial D_{x}^{-1}}{ }_{L} H^{(m, r)} s_{n}(x, y, z),
$$


where ( [19]; p. $32(8))$ :

$$
\frac{\partial}{\partial D_{x}^{-1}}:=\frac{\partial}{\partial x} x \frac{\partial}{\partial x} .
$$

Since, in view of Table $1(\mathrm{IV})$, we have

$$
{ }_{L} H_{n}^{(m, r)}(0, y, z)=H^{(r)}(y, z) .
$$

Therefore, from Example 4 of Section 3, we have

$$
{ }_{L} H^{(m, r)} s_{n}(0, y, z)={ }_{H^{(r)}} s_{n}(y, z) .
$$

Solving equation (4.7) subject to initial condition (4.9), we get assertion (4.6).

By making suitable substitutions for the indices and variables in operational representation (4.1), we find the operational representations for the special cases of the $\operatorname{LGHSP}_{L} H^{(m, r)} s_{n}(x, y, z)$.

\begin{tabular}{|c|c|c|c|c|}
\hline $\begin{array}{l}\text { S. } \\
\text { No. }\end{array}$ & $\begin{array}{l}\text { Values of } \\
\text { the Indices } \\
\text { and Variables }\end{array}$ & $\begin{array}{l}\text { Relation Between the } \\
\text { LGHSP }{ }^{L} H^{(m, r)} s_{n}(x, y, z) \\
\text { and its Special Case }\end{array}$ & $\begin{array}{l}\text { Name of the } \\
\text { Polynomials }\end{array}$ & $\begin{array}{l}\text { Operational Definition } \\
\text { of the Polynomials }\end{array}$ \\
\hline I. & $\begin{array}{l}m=1, \quad r=2 \\
x \rightarrow-x\end{array}$ & $\begin{array}{l}L^{H(1,2)} s_{n}(-x, y, z) \\
={ }_{L} H s_{n}(x, y, z)\end{array}$ & $\begin{array}{l}\text { 3-Variable } \\
\text { Laguerre -Hermite } \\
\text { based Sheffer }\end{array}$ & $\begin{array}{l}{ }_{L} H^{s_{n}}(x, y, z)=s_{n}\left(y-D_{x}^{-1}+2 z \frac{\partial}{\partial y}\right) \\
=\exp \left(-D_{x}^{-1} \frac{\partial}{\partial y}+z \frac{\partial^{2}}{\partial y^{2}}\right) s_{n}(y)\end{array}$ \\
\hline II. & $\begin{aligned} m & =1, r=2 \\
z & =-\frac{1}{2} \\
x & \rightarrow-x\end{aligned}$ & $\begin{array}{l}{ }_{L} H^{(1,2)} s_{n}\left(-x, y,-\frac{1}{2}\right) \\
={ }_{L} H^{*} s_{n}(x, y)\end{array}$ & $\begin{array}{l}\text { 2-Variable } \\
\text { Laguerre-Hermite } \\
\text { based Sheffer }\end{array}$ & $\begin{array}{l}L H^{\star} s_{n}(x, y)=s_{n}\left(y-D_{x}^{-1}-\frac{\partial}{\partial y}\right) \\
=\exp \left(-D_{x}^{-1} \frac{\partial}{\partial y}-\frac{1}{2} \frac{\partial^{2}}{\partial y^{2}}\right) s_{n}(y)\end{array}$ \\
\hline III. & $\begin{aligned} m & =1, \quad r=2 \\
y & =1, z \rightarrow y \\
x & \rightarrow-x\end{aligned}$ & $\begin{array}{l}L^{H^{(1,2)} s_{n}(-x, 1, y)} \\
=\varphi s_{n}(x, y)\end{array}$ & $\begin{array}{l}\text { Laguerre -Hermite } \\
\text { type based } \\
\text { Sheffer }\end{array}$ & $\begin{array}{l}\varphi^{s_{n}(x, y)}=s_{n}\left(1-D_{x}^{-1}-2 y \frac{\partial}{\partial x} x \frac{\partial}{\partial x}\right) \\
=\exp \left(y \frac{\partial^{2}}{\partial x^{2}} x^{2} \frac{\partial^{2}}{\partial x^{2}}-\frac{\partial}{\partial x} x \frac{\partial}{\partial x}\right) s_{n}\left(-D_{x}^{-1}\right)\end{array}$ \\
\hline IV. & $x=0$ & $\begin{array}{l}{ }_{L} H^{(m, r)}{ }^{s} n(0, y, z) \\
={ }_{H}(r) s_{n}(y, z)\end{array}$ & $\begin{array}{l}\text { Gould-Hopper } \\
\text { based Sheffer }\end{array}$ & $\begin{array}{l}H^{(r)} s_{n}(y, z)=s_{n}\left(y+r z \frac{\partial^{r-1}}{\partial y^{r-1}}\right) \\
=\exp \left(z \frac{\partial^{r}}{\partial y^{r}}\right) s_{n}(y)[35]\end{array}$ \\
\hline V. & $z=0$ & $\begin{array}{l}{ }_{L} H^{(m, r)}{ }^{s_{n}(x, y, 0)} \\
={ }_{m} L_{n} s_{n}(x, y)\end{array}$ & $\begin{array}{l}\text { 2-Variable } \\
\text { generalized Laguerre } \\
\text { based Sheffer }\end{array}$ & $\begin{array}{l}m L^{s_{n}(x, y)}=s_{n}\left(y+m D_{x}^{-1} \frac{\partial^{m-1}}{\partial y^{m-1}}\right) \\
=\exp \left(D_{x}^{-1} \frac{\partial^{m}}{\partial y^{m}}\right) s_{n}(y)\end{array}$ \\
\hline VI. & $\begin{array}{l}r=m ; x=0 \\
y \rightarrow-D_{x}^{-1} \\
z \rightarrow y\end{array}$ & $\begin{array}{l}L^{(m, m) s_{n}\left(0,-D_{x}^{-1}, y\right)} \\
={ }_{[m]} L s_{n}(x, y)\end{array}$ & $\begin{array}{l}\text { 2-Variable } \\
\text { generalized Laguerre } \\
\text { type based } \\
\text { Sheffer }\end{array}$ & $\begin{array}{l}{[m]^{L^{s_{n}}(x, y)}} \\
=s_{n}\left(-D_{x}^{-1}+(-1)^{m} m y \frac{\partial^{m-1}}{\partial x^{m-1}} x^{m-1} \frac{\partial^{m-1}}{\partial x^{m-1}}\right) \\
\quad=\exp \left(-(-1)^{m} y \frac{\partial^{m}}{\partial x^{m}} x^{m} \frac{\partial^{m}}{\partial x^{m}}\right) s_{n}\left(-D_{x}^{-1}\right)\end{array}$ \\
\hline VII. & $\begin{array}{l}r=m-1 ; x=0, \\
y \rightarrow x, z \rightarrow y\end{array}$ & $\begin{array}{l}L^{H}(m, m-1) s_{n}(0, x, y) \\
={ }_{U}(m) s_{n}(x, y)\end{array}$ & $\begin{array}{l}\text { Generalized Chebyshev } \\
\text { based Sheffer }\end{array}$ & $\begin{aligned} U^{(m)} s_{n}(x, y) & =s_{n}\left(x+(m-1) y \frac{\partial^{m-2}}{\partial x^{m-2}}\right) \\
= & \exp \left(y \frac{\partial^{m-1}}{\partial x^{m-1}}\right) s_{n}(x)\end{aligned}$ \\
\hline VIII. & $\begin{array}{l}m=1 ; z=0, \\
x \rightarrow-x\end{array}$ & $\begin{array}{l}L H^{(1, r)^{s_{n}(-x, y, 0)}} \\
={ }_{L} s_{n}(x, y)\end{array}$ & $\begin{array}{l}\text { 2-Variable Laguerre } \\
\text { based Sheffer }\end{array}$ & $\begin{array}{l}L^{s_{n}}(x, y)=s_{n}\left(y-D_{x}^{-1}\right) \\
=\exp \left(-D_{x}^{-1} \frac{\partial}{\partial y}\right) s_{n}(y) \\
=\exp \left(-y \frac{\partial}{\partial x} x \frac{\partial}{\partial x}\right) s_{n}\left(-D_{x}^{-1}\right)[32]\end{array}$ \\
\hline IX. & $\begin{array}{l}m=1 ; z=0 \\
x \rightarrow y \\
y \rightarrow-D_{x}^{-1}\end{array}$ & $\begin{array}{l}n !{ }_{L} H^{(1, r)} s_{n}\left(y,-D_{x}^{-1}, 0\right) \\
=R s_{n}(x, y)\end{array}$ & $\begin{array}{l}\text { 2-Variable Legendre } \\
\text { based Sheffer }\end{array}$ & $\begin{array}{l}\frac{R^{s} n(x, y)}{n !}=s_{n}\left(-D_{x}^{-1}+D_{y}^{-1}\right) \\
=\exp \left(-D_{x}^{-1} \frac{\partial}{\partial y} y \frac{\partial}{\partial y}\right) s_{n}\left(D_{y}^{-1}\right) \\
=\exp \left(-D_{y}^{-1} \frac{\partial}{\partial x} x \frac{\partial}{\partial x}\right) s_{n}\left(-D_{x}^{-1}\right)\end{array}$ \\
\hline
\end{tabular}
We mention these operational representations in Table 11.

Table 11. Operational representations for the special cases of the LGHSP $_{L} H^{(m, r)} s_{n}(x, y, z)$ 


\begin{tabular}{|c|c|c|c|c|}
\hline $\mathrm{X}$. & $\begin{array}{l}x=0, y \rightarrow x \\
z \rightarrow y D_{y} y\end{array}$ & $\begin{array}{l}L^{(m, r) s_{n}\left(0, x, y D_{y} y\right)} \\
=e^{(r) s_{n}(x, y)}\end{array}$ & $\begin{array}{l}\text { 2-Variable truncated } \\
\text { of order } r \\
\text { based Sheffer }\end{array}$ & $\begin{array}{l}e^{(r)} s_{n}(x, y)=s_{n}\left(x+r y \frac{\partial}{\partial y} y \frac{\partial^{r-1}}{\partial x^{r-1}}\right) \\
=\exp \left(y \frac{\partial}{\partial y} y \frac{\partial^{r}}{\partial x^{r}}\right) s_{n}(x)[36]\end{array}$ \\
\hline XI. & $r=2 ; x=0$ & $\begin{array}{l}L^{(m, 2)} s_{n}(0, y, z) \\
=H s_{n}(y, z)\end{array}$ & $\begin{array}{l}\text { 2-Variable Hermite- } \\
\text { Kampé de Fériet } \\
\text { based Sheffer }\end{array}$ & $\begin{array}{l}H^{s_{n}}(y, z)=s_{n}\left(y+2 z \frac{\partial}{\partial y}\right) \\
=\exp \left(z \frac{\partial^{2}}{\partial y^{2}}\right) s_{n}(y)[31]\end{array}$ \\
\hline XII. & $\begin{array}{l}r=2 ; x=0, \\
y \rightarrow D_{x}^{-1}, z \rightarrow y\end{array}$ & $\begin{array}{l}L_{H} H^{(m, 2)} s_{n}\left(0, D_{x}^{-1}, y\right) \\
=G s_{n}(x, y)\end{array}$ & $\begin{array}{l}\text { Hermite type } \\
\text { based Sheffer }\end{array}$ & 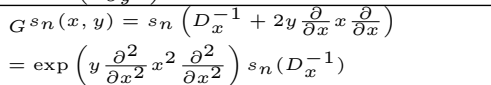 \\
\hline XIII. & $\begin{array}{l}m=2 ; z=0, \\
x \rightarrow\left(\frac{x^{2}-1}{4}\right)\end{array}$ & $\begin{array}{l}L^{(2, r)} s_{n}\left(\frac{x^{2}-1}{4}, x, 0\right) \\
={ }_{P} s_{n}(x)\end{array}$ & $\begin{array}{l}\text { Legendre } \\
\text { based Sheffer }\end{array}$ & $\begin{array}{l}P^{s n(x)}=s_{n}\left(x+2 D^{-1}\left(\frac{x^{2}-1}{4}\right)^{\frac{\partial}{\partial x}}\right. \\
=\exp \left(D^{-1}\left(\frac{x^{2}-1}{4}\right)^{\frac{\partial^{2}}{\partial x^{2}}}\right) s_{n}(x)\end{array}$ \\
\hline XIV. & $\begin{array}{l}x \rightarrow y D_{y} y \\
y \rightarrow x\end{array}$ & $\begin{array}{l}L^{(m, r)^{s}}{ }^{s_{n}\left(y D_{y} y, x, z\right)} \\
\left.=H^{(r, m)}\right)^{s_{n}(x, y, z)}\end{array}$ & $\begin{array}{l}\text { 3-Variable } \\
\text { generalized Hermite } \\
\text { based Sheffer }\end{array}$ & 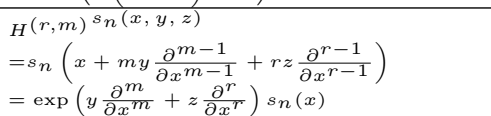 \\
\hline $\mathrm{XV}$. & $\begin{array}{l}m=2, r=3 \\
x \rightarrow z D_{z} z \\
y \rightarrow x, z \rightarrow y\end{array}$ & 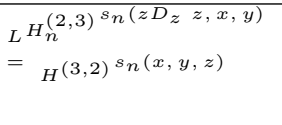 & based Sheffer & $\begin{array}{l}H^{(3,2)} s_{n}(x, y, z)=s_{n}\left(x+3 y \frac{\partial^{2}}{\partial x^{2}}+2 z \frac{\partial}{\partial x}\right) \\
=\exp \left(y \frac{\partial^{3}}{\partial x^{3}}+z \frac{\partial^{2}}{\partial x^{2}}\right) s_{n}(x)\end{array}$ \\
\hline
\end{tabular}

Next, we prove the integral representations for the LGHSP ${ }_{L} H^{(m, r)} s_{n}(x, y, z)$ in the form of following theorems:

Theorem 4.4. The following integral representation for the $L G H S P_{L} H^{(m, r)} s_{n}(x, y, z)$ holds true:

$$
{ }_{L} H^{(m, r)} s_{n}(x, y, z)=\frac{1}{2 \pi i} \int_{-\infty}^{(0+)} e^{u} u^{-1}{ }_{H^{(r, m)}} s_{n}\left(y, x u^{-1}, z\right) d u
$$

Proof. Using equation (1.13) in the l.h.s. of equation (2.1) and interchanging the sides, we have

$$
\sum_{n=0}^{\infty}{ }_{L} H^{(m, r)} s_{n}(x, y, z) \frac{t^{n}}{n !}=\frac{1}{g\left(f^{-1}(t)\right)} \sum_{n=0}^{\infty}{ }_{L} H_{n}^{(m, r)}(x, y, z) \frac{\left(f^{-1}(t)\right)^{n}}{n !} .
$$

Using the following integral representation of $\operatorname{LGHP}_{L} H_{n}^{(m, r)}(x, y, z)[29]$ :

$$
{ }_{L} H_{n}^{(m, r)}(x, y, z)=\frac{1}{2 \pi i} \int_{-\infty}^{(0+)} e^{u} u^{-1} H_{n}^{(r, m)}\left(y, x u^{-1}, z\right) d u,
$$

in the r.h.s. of equation (4.11), we find

$$
\sum_{n=0}^{\infty}{ }_{L} H^{(m, r)} s_{n}(x, y, z) \frac{t^{n}}{n !}=\frac{1}{2 \pi i} \frac{1}{g\left(f^{-1}(t)\right)} \int_{-\infty}^{(0+)} e^{u} u^{-1}\left(\sum_{n=0}^{\infty} H_{n}^{(r, m)}\left(y, x u^{-1}, z\right) \frac{\left(f^{-1}(t)\right)^{n}}{n !}\right) d u .
$$

Now, making use of the generating function equation of $H_{n}^{(r, m)}(x, y, z)$ given in Table 1 (XIV) in the r.h.s. of the above equation, we have

$$
\sum_{n=0}^{\infty}{ }_{L} H^{(m, r)} s_{n}(x, y, z) \frac{t^{n}}{n !}=\frac{1}{2 \pi i} \int_{-\infty}^{(0+)} e^{u} u^{-1}\left(\frac{1}{g\left(f^{-1}(t)\right)} e^{\left(y f^{-1}(t)+x u^{-1}\left(f^{-1}(t)\right)^{r}+z\left(f^{-1}(t)\right)^{m}\right)}\right) d u,
$$


which in view of generating function equation of ${ }_{H^{(r, m)}} s_{n}(x, y, z)$ given in Table 9 becomes

$$
\sum_{n=0}^{\infty}{ }_{L} H^{(m, r)} s_{n}(x, y, z) \frac{t^{n}}{n !}=\frac{1}{2 \pi i} \sum_{n=0}^{\infty}\left(\int_{-\infty}^{(0+)} e^{u} u_{H^{(r, m)}} s_{n}\left(y, x u^{-1}, z\right) d u\right) \frac{t^{n}}{n !} .
$$

Finally, equating the coefficients of like powers of $t$ in both sides of equation (4.14), we get assertion (4.10).

Theorem 4.5. The following integral representations for the $L G H S P_{L} H^{(m, r)} s_{n}(x, y, z)$ hold true:

$$
{ }_{L} H^{(m, r)} s_{n}(x, y, z)=\int_{0}^{\infty} e^{-u}{ }_{L} H^{(m, r)} s_{n}\left(x, y, u D_{z}^{-1}\right) d u
$$

and

$$
{ }_{L} H^{(m, r)} s_{n}(x, y, z)=\frac{1}{n !} \int_{0}^{\infty} e^{-u} u_{L}^{n} H^{(m+1, r)} s_{n}\left(\frac{x}{u}, y, z\right) d u
$$

Proof. Using the integral representations [29]:

$$
{ }_{L} H_{n}^{(m, r)}(x, y, z)=\int_{0}^{\infty} e^{-u}{ }_{L} H_{n}^{(m, r)}\left(x, y, u D_{z}^{-1}\right) d u
$$

and

$$
{ }_{L} H_{n}^{(m, r)}(x, y, z)=\frac{1}{n !} \int_{0}^{\infty} e^{-u} u^{n}{ }_{L} H_{n}^{(m+1, r)}\left(\frac{x}{u}, y, z\right) d u,
$$

respectively in the r.h.s. of equation (4.11), we find

$$
\sum_{n=0}^{\infty}{ }_{L} H^{(m, r)} s_{n}(x, y, z) \frac{t^{n}}{n !}=\frac{1}{g\left(f^{-1}(t)\right)} \sum_{n=0}^{\infty}\left(\int_{0}^{\infty} e^{-u}{ }_{L} H_{n}^{(m, r)}\left(x, y, u D_{z}^{-1}\right) d u\right) \frac{\left(f^{-1}(t)\right)^{n}}{n !}
$$

or, equivalently

$$
\sum_{n=0}^{\infty}{ }_{L} H^{(m, r)} s_{n}(x, y, z) \frac{t^{n}}{n !}=\int_{0}^{\infty} e^{-u}\left(\frac{1}{g\left(f^{-1}(t)\right)} \sum_{n=0}^{\infty}{ }_{L} H_{n}^{(m, r)}\left(x, y, u D_{z}^{-1}\right) \frac{\left(f^{-1}(t)\right)^{n}}{n !}\right) d u
$$

and

$$
\sum_{n=0}^{\infty}{ }_{L} H^{(m, r)} s_{n}(x, y, z) \frac{t^{n}}{n !}=\frac{1}{g\left(f^{-1}(t)\right)} \sum_{n=0}^{\infty}\left(\frac{1}{n !} \int_{0}^{\infty} e^{-u} u^{n}{ }_{L} H_{n}^{(m+1, r)}\left(\frac{x}{u}, y, z\right) d u\right) \frac{\left(f^{-1}(t)\right)^{n}}{n !},
$$

or, equivalently

$$
\sum_{n=0}^{\infty}{ }_{L} H^{(m, r)} s_{n}(x, y, z) \frac{t^{n}}{n !}=\frac{1}{n !} \int_{0}^{\infty} e^{-u} u^{n}\left(\frac{1}{g\left(f^{-1}(t)\right)} \sum_{n=0}^{\infty}{ }_{L} H_{n}^{(m+1, r)}\left(\frac{x}{u}, y, z\right) \frac{\left(f^{-1}(t)\right)^{n}}{n !}\right) d u
$$


respectively.

Again, using equation (4.11) in the r.h.s. of equations (4.19) and (4.20), we find

$$
\sum_{n=0}^{\infty}{ }_{L} H^{(m, r)} s_{n}(x, y, z) \frac{t^{n}}{n !}=\sum_{n=0}^{\infty}\left(\int_{0}^{\infty} e^{-u}{ }_{L} H^{(m, r)} s_{n}\left(x, y, u D_{z}^{-1}\right) d u\right) \frac{t^{n}}{n !}
$$

and

$$
\sum_{n=0}^{\infty}{ }_{L} H^{(m, r)} s_{n}(x, y, z) \frac{t^{n}}{n !}=\sum_{n=0}^{\infty}\left(\frac{1}{n !} \int_{0}^{\infty} e^{-u} u_{L}^{n} H^{(m+1, r)} s_{n}\left(\frac{x}{u}, y, z\right) d u\right) \frac{t^{n}}{n !}
$$

respectively. Finally, equating the coefficients of like powers of $t$ in both sides of equations (4.21) and (4.22), we get assertions (4.15) and (4.16), respectively.

In Section 3, we have obtained the results for the new and known families of special polynomials related to Sheffer sequences by taking the special cases of the $\operatorname{LGHP}{ }_{L} H_{n}^{(m, r)}(x, y, z)$. In the Appendix section, we consider certain special polynomials belonging to the Sheffer and associated Sheffer families and obtain the results for the corresponding mixed special polynomials.

\section{Appendix}

The Sheffer class contains important sequences such as the Hermite, Laguerre, Bernoulli, PoissonCharlier polynomials etc. These polynomials are important from the view point of applications in physics and number theory. Also, the associated Sheffer family contains Mittag-Leffler, exponential, lower factorial polynomials etc.

We present the lists of some known members of the Sheffer and associated Sheffer families in Tables 12 and 13 respectively.

\begin{tabular}{|c|c|c|c|c|}
\hline s. No. & $g(t) ; A(t)$ & $f(t) ; H(t)$ & Generating Functions & Polynomials \\
\hline I. & $e^{\left(\frac{t}{\nu}\right)^{k}} ; e^{-t^{k}}$ & $\frac{t}{\nu} ; \nu t$ & $\begin{array}{l}\exp \left(\nu x t-t^{k}\right) \\
=\sum_{n=0}^{\infty} H_{n, k, \nu}(x) \frac{t^{n}}{n !}\end{array}$ & $\begin{array}{l}\text { Generalized Hermite } \\
\text { polynomials } \\
H_{n, k, \nu}(x)[38]\end{array}$ \\
\hline II. & $(1-t)^{-\alpha-1} ;(1-t)^{-\alpha-1}$ & $\frac{t}{t-1} ; \quad \frac{t}{t-1}$ & $\begin{array}{l}\frac{1}{(1-t)^{\alpha+1}} \exp \left(\frac{x t}{t-1}\right) \\
=\sum_{n=0}^{\infty} L_{n}^{(\alpha)}(x) t^{n}\end{array}$ & $\begin{array}{l}\text { Generalized Laguerre } \\
\text { polynomials } \\
n ! L_{n}^{\alpha}(x)[1,39] \\
\end{array}$ \\
\hline III. & $\frac{2}{e^{t}-1} ; \quad \frac{t}{1-t}$ & $\frac{e^{t}-1}{e^{t}+1} ; \ln \left(\frac{1+t}{1-t}\right)$ & $\begin{array}{l}\frac{t}{1-t}\left(\frac{1+t}{1-t}\right)^{x} \\
=\sum_{n=0}^{\infty} P_{n}(x) \frac{t^{n}}{n !}\end{array}$ & $\begin{array}{l}\text { Pidduck polynomials } \\
P_{n}(x)[7,26]\end{array}$ \\
\hline IV. & $(1-t)^{-\beta} ; e^{\beta t}$ & $\ln (1-t) ; 1-e^{t}$ & $\begin{array}{l}\exp \left(\beta t+x\left(1-e^{t}\right)\right) \\
=\sum_{n=0}^{\infty} a_{n}^{(\beta)}(x) \frac{t^{n}}{n !}\end{array}$ & $\begin{array}{l}\text { Acturial polynomials } \\
a_{n}^{(\beta)}(x)[7]\end{array}$ \\
\hline
\end{tabular}

Table 12. Some members of the Sheffer family 


\begin{tabular}{|c|c|c|c|c|}
\hline V. & $\exp \left(a\left(e^{t}-1\right)\right) ; e^{-t}$ & $a\left(e^{t}-1\right) ; \ln \left(1+\frac{t}{a}\right)$ & $\begin{array}{l}e^{-t}\left(1+\frac{t}{a}\right)^{x} \\
=\sum_{n=0}^{\infty} c_{n}(x ; a) \frac{t^{n}}{n !}\end{array}$ & $\begin{array}{l}\text { Poisson-Charlier } \\
\text { polynomials } \\
c_{n}(x ; a)[25,28,42]\end{array}$ \\
\hline VI. & $\left(1+e^{\lambda t}\right)^{\mu} ;\left(1+(1+t)^{\lambda}\right)^{-\mu}$ & $e^{t}-1 ; \ln (1+t)$ & $\begin{array}{l}\left(1+(1+t)^{\lambda}\right)^{-\mu}(1+t)^{x} \\
=\sum_{n=0}^{\infty} S_{n}(x ; \lambda, \mu) \frac{t^{n}}{n !}\end{array}$ & $\begin{array}{l}\text { Peters polynomials } \\
S_{n}(x ; \lambda, \mu)[7]\end{array}$ \\
\hline VII. & $\frac{t}{e^{t}-1} ; \quad \frac{t}{\ln (1+t)}$ & $e^{t}-1 ; \ln (1+t)$ & $\begin{array}{l}\frac{t}{\ln (1+t)}(1+t)^{x} \\
=\sum_{n=0}^{\infty} b_{n}(x) \frac{t^{n}}{n !}\end{array}$ & $\begin{array}{l}\text { Bernoulli polynomials } \\
\text { of the second kind } \\
b_{n}(x)[28]\end{array}$ \\
\hline VIII. & $\frac{1}{2}\left(1+e^{t}\right) ; \frac{2}{2+t}$ & $e^{t}-1 ; \ln (1+t)$ & $\begin{array}{l}\frac{2}{2+t}(1+t)^{x} \\
=\sum_{n=0}^{\infty} r_{n}(x) \frac{t^{n}}{n !}\end{array}$ & $\begin{array}{l}\text { Related polynomials } \\
r_{n}(x)[28]\end{array}$ \\
\hline IX. & $\sec t ; \frac{1}{\sqrt{1+t^{2}}}$ & $\tan t ; \arctan (t)$ & $\begin{array}{l}\frac{1}{\sqrt{1+t^{2}}} \exp (x \arctan (t)) \\
=\sum_{n=0}^{\infty} R_{n}(x) \frac{t^{n}}{n !}\end{array}$ & $\begin{array}{l}\text { Hahn polynomials } \\
R_{n}(x)[6]\end{array}$ \\
\hline $\mathrm{X}$. & $\frac{1+t}{(1-t)^{a}} ; \quad(1-4 t)^{-\frac{1}{2}}\left(\frac{2}{1+\sqrt{1-4 t}}\right)^{a-1}$ & $\frac{1}{4}-\frac{1}{4}\left(\frac{1+t}{1-t}\right)^{2} ; \quad \frac{-4 t}{(1+\sqrt{1-4 t})^{2}}$ & $\begin{array}{l}(1-4 t)^{-\frac{1}{2}}\left(\frac{2}{1+\sqrt{1-4 t}}\right)^{a-1} \\
\times \exp \left(\frac{-4 x t}{(1+\sqrt{1-4 t})^{2}}\right) \\
=\sum_{n=0}^{\infty} R_{n}(a, x) t^{n}\end{array}$ & $\begin{array}{l}\text { Shively's psedo-Laguerre } \\
\text { polynomials } \\
R_{n}(a, x)[39]\end{array}$ \\
\hline
\end{tabular}

Table 13. Some members of the associated Sheffer family

\begin{tabular}{llll}
\hline S. No. & $f(t) ; H(t)$ & Generating Functions & Polynomials \\
\hline I. & $\frac{e^{t}-1}{e^{t}+1} ; \ln \left(\frac{1+t}{1-t}\right)$ & $\left(\frac{1+t}{1-t}\right)^{x}=\sum_{n=0}^{\infty} M_{n}(x) \frac{t^{n}}{n !}$ & Mittag-Leffler polynomials $M_{n}(x)[4]$ \\
\hline II. & $\ln (1+t) ; e^{t}-1$ & $\exp \left(x\left(e^{t}-1\right)\right)=\sum_{n=0}^{\infty} \varphi_{n}(x) \frac{t^{n}}{n !}$ & Exponential polynomials $\varphi_{n}(x)[5]$ \\
\hline III. & $e^{t}-1 ; \ln (1+t)$ & $(1+t)^{x}=\sum_{n=0}^{\infty}(x)_{n} \frac{t^{n}}{n !}$ & Lower factorial polynomials $(x)_{n}[40]$ \\
\hline IV. & $-\frac{1}{2} t^{2}+t ; 1-\sqrt{1-2 t}$ & $\exp (x(1-\sqrt{1-2 t}))=\sum_{n=0}^{\infty} p_{n}(x) \frac{t^{n}}{n !}$ & Bessel polynomials $p_{n}(x)[8,37]$ \\
\hline
\end{tabular}

Remark 5.1. We remark that corresponding to each member belonging to the Sheffer (or associated Sheffer) family, there exists a new special polynomial belonging to the LGHSP (or LGHASP) family. The generating function and other properties of these special polynomials can be obtained from the results derived in Section 2.

Thus, by taking $g(t)$ (or $A(t)$ ) and $f(t)$ (or $H(t)$ ) of the special polynomials belonging to Sheffer family (Table 12 (I to X)) in equations (2.1) (or (2.2)), (2.8a) (or (2.8b)) and (2.9a) (or (2.9b)), we get the generating function, multiplicative and derivative operators for the corresponding members belonging to the LGHSP family.

We present these results along with the name and notation of the resultant special polynomial belonging to the LGHSP family in Table 14 .

Table 14. Certain results for the members belonging to the LGHSP family 


\begin{tabular}{|c|c|c|c|}
\hline s. No. & $\begin{array}{l}\text { Name/Notation of the } \\
\text { Resultant Special } \\
\text { Polynomial }\end{array}$ & Generating Function & Multiplicative and Derivative Operators \\
\hline I. & $\begin{array}{l}{ }_{L} H^{(m, r)}{ }^{H} n, k, \nu(x, y, z):= \\
\text { Laguerre- } \\
\text { Gould Hopper based } \\
\text { generalized Hermite } \\
\text { polynomials }\end{array}$ & $\begin{array}{l}e^{-t^{k}} C_{0}\left(-x(\nu t)^{m}\right) \exp \left(y \nu t+z(\nu t)^{r}\right) \\
=\sum_{n=0}^{\infty} H^{(m, r)} H_{n, k, \nu}(x, y, z) \frac{t^{n}}{n !}\end{array}$ & $\begin{aligned} \hat{M}= & \nu\left(y+m D_{x}^{-1} \frac{\partial^{m-1}}{\partial y^{m-1}}+r z \frac{\partial^{r-1}}{\partial y^{r-1}}\right) \\
& \quad-k\left(\frac{1}{\nu} \partial y\right)^{k-1} \\
\hat{P}= & \frac{1}{\nu} \partial y\end{aligned}$ \\
\hline II. & $\begin{array}{l}n ! \\
\quad{ }_{L} H(m, r) \\
\text { Laguerre- } \\
\\
\text { Gould Hopper based } \\
\text { generalized Laguerre } \\
\text { polynomials }\end{array}$ & $\begin{array}{l}(1-t)^{-\alpha-1} C_{0}\left(-x\left(\frac{t}{t-1}\right)^{m}\right) \exp \left(\frac{y t}{t-1}+z\left(\frac{t}{t-1}\right)^{r}\right) \\
=\sum_{n=0}^{\infty} L^{(m, r)} L_{n}^{(\alpha)}(x, y, z) t^{n}\end{array}$ & $\begin{aligned} \hat{M}=- & \left(y+m D_{x}^{-1} \frac{\partial^{m-1}}{\partial y^{m-1}}+r z \frac{\partial^{r-1}}{\partial y^{r-1}}\right) \\
& \quad(\partial y-1)^{2}+(a+1)(\partial y-1), \\
\hat{P}= & \frac{\partial y}{\partial y-1}\end{aligned}$ \\
\hline III. & $\begin{array}{l}{ }_{L} H^{(m, r)} P_{n}(x, y, z):= \\
\text { Laguerre- } \\
\text { Gould Hopper based } \\
\text { Pidduck polynomials }\end{array}$ & $\begin{array}{l}\frac{t}{1-t} C_{0}\left(-x\left(\ln \left(\frac{1+t}{1-t}\right)\right)^{m}\right) \times \\
\exp \left(y \ln \left(\frac{1+t}{1-t}\right)+z\left(\ln \left(\frac{1+t}{1-t}\right)\right)^{r}\right) \\
=\sum_{n=0}^{\infty} H^{(m, r)} P_{n}(x, y, z) \frac{t^{n}}{n !}\end{array}$ & $\begin{aligned} \hat{M}= & \left(y+m D_{x}^{-1} \frac{\partial^{m-1}}{\partial y^{m-1}}+r z \frac{\partial^{r-1}}{\partial y^{r-1}}\right) \\
& \left(\frac{\left(e^{\partial y+1)^{2}}\right.}{2 e^{\partial y}}\right)+\frac{\left(e^{\partial y}+1\right)^{2}}{2\left(e^{\partial y}-1\right)} \\
\hat{P}= & \frac{e^{\partial y}-1}{e^{\partial y}+1}\end{aligned}$ \\
\hline IV. & $\begin{array}{l}{ }_{L} H^{(m, r)} a_{n}^{(\beta)}(x, y, z):= \\
\text { Laguerre- } \\
\text { Gould Hopper based } \\
\text { Acturial polynomials } \\
\end{array}$ & $\begin{array}{l}e^{\beta t} C_{0}\left(-x\left(1-e^{t}\right)^{m}\right) \exp \left(y\left(1-e^{t}\right)+z\left(1-e^{t}\right)^{r}\right) \\
=\sum_{n=0}^{\infty} H_{H}^{(m, r)} a_{n}^{(\beta)}(x, y, z) \frac{t^{n}}{n !}\end{array}$ & $\begin{aligned} & \hat{M}=-\left(y+m D_{x}^{-1} \frac{\partial^{m-1}}{\partial y^{m-1}}+r z \frac{\partial^{r-1}}{\partial y^{r-1}}\right) \\
& \quad\left(1-\partial_{y}\right)+\beta \\
& \hat{P}=\ln \left(1-\partial_{y}\right)\end{aligned}$ \\
\hline V. & $\begin{array}{l}{ }_{L} H^{(m, r)}{ }^{c_{n}}(x, y, z ; a):= \\
\text { Laguerre- } \\
\text { Gould Hopper based } \\
\text { Poisson-Charlier } \\
\text { polynomials }\end{array}$ & $\begin{array}{l}e^{-t} C_{0}\left(-x\left(\ln \left(1+\frac{t}{a}\right)\right)^{m}\right) \times \\
\exp \left(y \ln \left(1+\frac{t}{a}\right)+z\left(\ln \left(1+\frac{t}{a}\right)\right)^{r}\right) \\
=\sum_{n=0}^{\infty} L_{H}^{(m, r)}{ }^{c_{n}(x, y, z ; a) \frac{t^{n}}{n !}}\end{array}$ & $\begin{aligned} \hat{M}= & a^{-1}\left(y+m D_{x}^{-1} \frac{\partial^{m-1}}{\partial y^{m-1}}+r z \frac{\partial^{r-1}}{\partial y^{r-1}}\right) \\
& \left(e^{-\partial y}\right)-1 \\
\hat{P}= & a\left(e^{\partial y}-1\right)\end{aligned}$ \\
\hline VI. & $\begin{array}{l}{ }_{L} H^{(m, r)} S_{n}(x, y, z ; \lambda, \mu):= \\
\text { Laguerre- } \\
\text { Gould Hopper based } \\
\text { Peters polynomials }\end{array}$ & $\begin{array}{l}\left(1+(1+t)^{\lambda}\right)^{-\mu} C_{0}\left(-x(\ln (1+t))^{m}\right) \times \\
\exp \left(y \ln (1+t)+z(\ln (1+t))^{r}\right) \\
=\sum_{n=0}^{\infty}{ }_{0} H^{(m, r)} S_{n}(x, y, z ; \lambda, \mu) \frac{t^{n}}{n !}\end{array}$ & $\begin{aligned} \hat{M}= & \left(y+m D_{x}^{-1} \frac{\partial^{m-1}}{\partial y^{m-1}}+r z \frac{\partial^{r-1}}{\partial y^{r-1}}\right) \\
& \left(e^{-\partial_{y}}\right)-\lambda \mu\left(1+e^{\lambda \partial y}\right)^{-1} e^{(\lambda-1) \partial y} \\
\hat{P}= & e^{\partial y}-1\end{aligned}$ \\
\hline VII. & $\begin{array}{l}L^{H}(m, r) b_{n}(x, y, z):= \\
\text { Laguerre- } \\
\text { Gould Hopper based } \\
\text { Bernoulli polynomials } \\
\text { of the second kind }\end{array}$ & $\begin{array}{l}\frac{t}{\ln (1+t)} C_{0}\left(-x(\ln (1+t))^{m}\right) \exp \left(y \ln (1+t)+z(\ln (1+t))^{r}\right) \\
=\sum_{n=0}^{\infty} L^{(m, r)} b_{n}(x, y, z) \frac{t^{n}}{n !}\end{array}$ & $\begin{aligned} \hat{M}= & \left(y+m D_{x}^{-1} \frac{\partial^{m-1}}{\partial y^{m-1}}+r z \frac{\partial^{r-1}}{\partial y^{r-1}}\right) \\
& \left(e^{-\partial y}\right)+\frac{\partial y}{e^{\partial y}-1}-\frac{1}{e^{\partial y}} \\
\hat{P}= & e^{\partial y}-1\end{aligned}$ \\
\hline VIII. & $\begin{array}{l}{ }_{L} H^{(m, r)} r_{n}(x, y, z):= \\
\text { Laguerre- } \\
\text { Gould Hopper based } \\
\text { Related polynomials } \\
\end{array}$ & $\begin{array}{l}\frac{2}{2+t} C_{0}\left(-x(\ln (1+t))^{m}\right) \exp \left(y \ln (1+t)+z(\ln (1+t))^{r}\right) \\
=\sum_{n=0}^{\infty}{ }_{L} H^{(m, r)^{r}} r_{n}(x, y, z) \frac{t^{n}}{n !}\end{array}$ & $\begin{aligned} \hat{M}= & \left(y+m D_{x}^{-1} \frac{\partial^{m-1}}{\partial y^{m-1}}+r z \frac{\partial^{r-1}}{\partial y^{r-1}}\right) \\
& \left(e^{-\partial y}\right)-\left(e^{\partial y}+1\right)^{-1} \\
\hat{P}= & e^{\partial y}-1\end{aligned}$ \\
\hline IX. & $\begin{array}{l}{ }_{L} H^{(m, r)} R_{n}(x, y, z):= \\
\text { Laguerre- } \\
\text { Gould Hopper based } \\
\text { Hahn polynomials } \\
\end{array}$ & $\begin{array}{l}\frac{1}{\sqrt{1+t^{2}}} C_{0}\left(-x(\arctan (t))^{m}\right) \exp \left(y \arctan (t)+z(\arctan (t))^{r}\right) \\
=\sum_{n=0}^{\infty} H^{(m, r)} R_{n}(x, y, z) \frac{t^{n}}{n !}\end{array}$ & $\begin{aligned} \hat{M}= & \left(y+m D_{x}^{-1} \frac{\partial^{m-1}}{\partial y^{m-1}}+r z \frac{\partial^{r-1}}{\partial y^{r-1}}\right) \\
& \left(\cos ^{2}\left(\partial_{y}\right)\right)-\cos \left(\partial_{y}\right) \sin \left(\partial_{y}\right), \\
\hat{P}= & \tan \left(\partial_{y}\right)\end{aligned}$ \\
\hline $\mathrm{x}$ & $\begin{array}{l}{ }_{L} H^{(m, r)} R_{n}(a, x, y, z):= \\
\text { Laguerre- } \\
\text { Gould Hopper based } \\
\text { Shively's } \\
\text { pseudo-Laguerre } \\
\text { polynomials }\end{array}$ & $\begin{array}{l}(1-4 t)^{-\frac{1}{2}}\left(\frac{2}{1+\sqrt{1-4 t}}\right)^{a-1} C_{0}\left(-x\left(\frac{-4 t}{(1+\sqrt{1-4 t})^{2}}\right)^{m}\right) \times \\
\exp \left(\frac{-4 y t}{(1+\sqrt{1-4 t})^{2}}+z\left(\frac{-4 t}{(1+\sqrt{1-4 t})^{2}}\right)^{r}\right) \\
=\sum_{n=0}^{\infty} H^{(m, r)} R_{n}(a, x, y, z) t^{n}\end{array}$ & $\begin{array}{c}\hat{M}=-\left(y+m D_{x}^{-1} \frac{\partial^{m-1}}{\partial y^{m-1}}+r z \frac{\partial^{r-1}}{\partial y^{r-1}}\right) \\
\left(\frac{\left(1-\partial_{y}\right)^{3}}{(1+\partial y)}+\left(\frac{1-\partial_{y}}{1+\partial y}\right)^{2}\right. \\
\quad(2+(a-1)(1+\partial y)) \\
\hat{P}=\frac{1}{4}-\frac{1}{4}\left(\frac{1+\partial_{y}}{1-\partial y}\right)^{2}\end{array}$ \\
\hline
\end{tabular}


Similarly, by taking $g(t)$ (or $A(t)$ ) and $f(t)$ (or $H(t)$ ) of the special polynomials belonging to the associated Sheffer family (Table 13 (I to IV)) in appropriate equations, we get the corresponding results for the members belonging to the LGHASP family.

We present these results along with the name and notation of the resultant special polynomial belonging to the LGHASP family in in Table 15.

Table 15. Certain results for the members belonging to the LGHASP family

\begin{tabular}{|c|c|c|c|}
\hline s. No. & $\begin{array}{l}\text { Name/Notation of the } \\
\text { Resultant Special } \\
\text { Polynomial }\end{array}$ & Generating Function & Multiplicative and Derivative Operators \\
\hline I. & $\begin{array}{l}{ }_{L} H^{(m, r)} M_{n}(x, y, z):= \\
\text { Laguerre- } \\
\text { Gould Hopper based } \\
\text { Mittag-Leffler } \\
\text { polynomials }\end{array}$ & $\begin{array}{l}C_{0}\left(-x\left(\ln \left(\frac{1+t}{1-t}\right)\right)^{m}\right) \exp \left(y \ln \left(\frac{1+t}{1-t}\right)+z\left(\ln \left(\frac{1+t}{1-t}\right)\right)^{r}\right) \\
=\sum_{n=0}^{\infty}{ }_{L} H^{(m, r)}{ }^{M_{n}(x, y, z) \frac{t^{n}}{n !}}\end{array}$ & $\begin{array}{l}\hat{M}=\left(y+m D_{x}^{-1} \frac{\partial^{m-1}}{\partial y^{m-1}}+r z \frac{\partial^{r-1}}{\partial y^{r-1}}\right) \\
\quad\left(\frac{\left(1+e^{\partial y}\right)^{2}}{2 e^{\partial y}}\right), \\
\hat{P}=\frac{e^{\partial y}-1}{e^{\partial y}+1}\end{array}$ \\
\hline II. & $\begin{array}{l}{ }_{L} H^{(m, r)} \varphi_{n}(x, y, z):= \\
\text { Laguerre- } \\
\text { Gould Hopper based } \\
\text { Exponential } \\
\text { polynomials } \\
\end{array}$ & $\begin{array}{l}C_{0}\left(-x\left(e^{t}-1\right)^{m}\right) \exp \left(y\left(e^{t}-1\right)+z\left(e^{t}-1\right)^{r}\right) \\
=\sum_{n=0}^{\infty}{ }_{L} H^{(m, r)} \varphi_{n}(x, y, z) \frac{t^{n}}{n !}\end{array}$ & $\begin{aligned} \hat{M}= & \left(y+m D_{x}^{-1} \frac{\partial^{m-1}}{\partial y^{m-1}}+r z \frac{\partial^{r-1}}{\partial y^{r-1}}\right) \\
& \left(1+\partial_{y}\right) \\
\hat{P}= & \ln \left(1+\partial_{y}\right)\end{aligned}$ \\
\hline III. & $\begin{array}{l}{ }_{L} H^{(m, r)}(x, y, z)_{n}:= \\
\text { Laguerre- } \\
\text { Gould Hopper based } \\
\text { Lower factorial } \\
\text { polynomials }\end{array}$ & $\begin{array}{l}C_{0}\left(-x(\ln (1+t))^{m}\right) \exp \left(y \ln (1+t)+z(\ln (1+t))^{r}\right) \\
=\sum_{n=0}^{\infty}{ }_{L} H^{(m, r)}(x, y, z) n \frac{t^{n}}{n !}\end{array}$ & $\begin{aligned} \hat{M}= & \left(y+m D_{x}^{-1} \frac{\partial^{m-1}}{\partial y^{m-1}}+r z \frac{\partial^{r-1}}{\partial y^{r-1}}\right) \\
& \left(e^{-\partial y}\right) \\
\hat{P}= & e^{\partial y}-1\end{aligned}$ \\
\hline IV. & $\begin{array}{l}L_{H} H^{(m, r)} p_{n}(x, y, z):= \\
\text { Laguerre- } \\
\text { Gould Hopper based } \\
\text { Bessel polynomials }\end{array}$ & $\begin{array}{l}C_{0}\left(-x(1-\sqrt{1-2 t})^{m}\right) \times \\
\exp \left(y(1-\sqrt{1-2 t})+z(1-\sqrt{1-2 t})^{r}\right) \\
=\sum_{n=0}^{\infty}{ }_{L} H^{(m, r)} p_{n}(x, y, z) \frac{t^{n}}{n !}\end{array}$ & $\begin{array}{l}\hat{M}=\left(y+m D_{x}^{-1} \frac{\partial^{m-1}}{\partial y^{m-1}}+r z \frac{\partial^{r-1}}{\partial y^{r-1}}\right) \frac{1}{1-\partial_{y}} \\
\hat{P}=-\frac{1}{2} \partial_{y}^{2}+\partial_{y}\end{array}$ \\
\hline
\end{tabular}

\section{References}

[1] L. C. Andrews, Special Functions for Engineers and Applied Mathematicians, Macmillan Publishing Company, New York, 1985.

[2] P. Appell, Sur une classe de polynômes, Ann. Sci. École. Norm. Sup. 9(2) (1880), 119-144.

[3] P. Appell and J. Kampé de Fériet, Fonctions hypergéométriques et hypersphériques: Polynômes d'Hermite, Gauthier-Villars, Paris, 1926.

[4] H. Bateman, The polynomial of Mittag-Leffler, Proc. Natl. Acad. Sci. U.S.A. 26 (1940), 491496.

[5] E. T. Bell, Exponential polynomials, Ann. Math. 35 (1934), 258-277. 
[6] C. M. Bender, Solution of operator equations of motion, J. Dittrich, P. Exner (Eds.), Rigorous Results in Quantum Dynamics, World Scientific, Singapore, (1991), 99-112.

[7] R. P. Boas and R. C. Buck, Polynomial Expansions of Analytic Functions, Springer-Verlag, Berlin, Gottingen, Heidelberg, 1958.

[8] L. Carlitz, A note on the Bessel polynomials, Duke Math. J. 24 (1957), 151-162.

[9] G. Dattoli, Hermite-Bessel and Laguerre-Bessel functions: A by-product of the monomiality principle, Advanced Special Functions and Applications (Melfi, 1999), 147-164, Proc. Melfi Sch. Adv. Top. Math. Phys., 1, Aracne, Rome, 2000.

[10] G. Dattoli, Integral transforms and Chebyshev-like polynomials, Appl. Math. Comput. 148 (2004), 225-234.

[11] G. Dattoli, C. Cesarano and D. Sacchetti, A note on truncated polynomials, Appl. Math. Comput. 134 (2003), 595-605.

[12] G. Dattoli, B. Germano, M.R. Martinelli and P.E. Ricci, Monomiality, orthogonal and pseudoorthogonal polynomials, Int. Math. Forum 1(13-16) (2006), 603-616.

[13] G. Dattoli, S. Lorenzutta, A.M. Mancho and A. Torre, Generalized polynomials and associated operational identities, J. Comput. Appl. Math. 108(1-2) (1999), 209-218.

[14] G. Dattoli, M. Migliorati and H.M. Srivastava, A class of Bessel summation formulas and associated operational methods, Fract. Calc. Appl. Anal. 7(2) (2004), 169-176.

[15] G. Dattoli, M. Migliorati and H. M. Srivastava, Sheffer polynomials, monomiality principle, algebraic methods and the theory of classical polynomials, Math. Comput. Modelling 45(9-10) (2007), 1033-1041.

[16] G. Dattoli, P.L. Ottaviani, A. Torre and L. Vázquez, Evolution operator equations: integration with algebraic and finite-difference methods. Applications to physical problems in classical and quantum mechanics and quantum field theory, Riv. Nuovo Cimento Soc. Ital. Fis. 20(2) (1997), $1-133$.

[17] G. Dattoli, P.E. Ricci and C. Cesarano, A note on Legendre polynomials, Int. J. Nonlinear Sci. Numer. Simul. 2(4) (2001), 365-370..

[18] G. Dattoli, P.E. Ricci and I. Khomasuridze, On the derivation of new families of generating functions involving ordinary Bessel functions and Bessel-Hermite functions, Math. Comput. Modelling 46 (2007), 410-414.

[19] G. Dattoli, H.M. Srivastava and K. Zhukovsky, A new family of integral transforms and their applications, Integral Transforms Spec. Funct. 17(1) (2006), 31-37.

[20] G. Dattoli and A. Torre, Operational methods and two variable Laguerre polynomials, Atti Acad. Sci. Torino Cl. Sci. Fis. Mat. Natur. 132 (1998), 1-7.

[21] G. Dattoli and A. Torre, Operational identities and properties of ordinary and generalized special functions, J. Math. Anal. Appl. 236 (1999), 399-414. 
[22] G. Dattoli and A. Torre, Exponential operators, quasi-monomials and generalized polynomials, Radiat. Phys. Chem. 57(1) (2000), 21-26.

[23] G. Dattoli, A. Torre, S. Lorenzutta and C. Cesarano, Generalized polynomials and operational identities, Atti. Acad. Sci. Torino Cl. Sci. Fis. Mat. Natur. 134 (2000), 231-249.

[24] G. Dattoli, A. Torre and A.M. Mancho, The generalized Laguerre polynomials, the associated Bessel functions and applications to propagation problems, Radiat. Phys. Chem. 59 (2000), 229-237.

[25] A. Erdélyi, W. Magnus, F. Oberhettinger and F. G. Tricomi, Higher Transcendental Functions, The Bateman Manuscript Project, Vol. II, McGraw-Hill, New York, Toronto, London, 1953.

[26] A. Erdélyi, W. Magnus, F. Oberhettinger and F. G. Tricomi, Higher Transcendental Functions, The Bateman Manuscript Project, Vol. III, McGraw-Hill, New York, Toronto, London, 1955.

[27] H.W. Gould and A.T. Hopper, Operational formulas connected with two generalizations of Hermite polynomials, Duke. Math. J. 29 (1962), 51-63.

[28] C. Jordan, Calculas of Finite Differences, Third Ed., Chelsea, Bronx, New York, 1965.

[29] Subuhi Khan and Ahmed Ali Al-Gonah, Operational methods and Laguerre-Gould Hopper polynomials, Appl. Math. Comput. 218 (2012), 9930-9942.

[30] Subuhi Khan and Mahvish Ali, Certain families associated with Chebyshev and Sheffer polynomials, Accepted in the proceeding "CSI Transactions on ICT", being published by Springer.

[31] Subuhi Khan, M.W.M. Al-Saad and Ghazala Yasmin, Some properties of Hermite-based Sheffer polynomials, Appl. Math. Comput. 217(5) (2010), 2169-2183.

[32] Subuhi Khan and Nusrat Raza, Monomiality principle, operational methods and family of Laguerre-Sheffer polynomials, J. Math. Anal. Appl. 387 (2012), 90-102.

[33] Subuhi Khan and Nusrat Raza, Families of Legendre-Sheffer polynomials, Math. Comput. Modelling 55 (2012), 969-982.

[34] Subuhi Khan, Nusrat Raza and Mahvish Ali, Finding mixed families of special polynomials associated with Appell sequences, Preprint.

[35] Subuhi Khan and Mumtaz Riyasat, Determinantal approach to certain mixed special polynomials related to Gould-Hopper polynomials, Appl. Math. Comput. 251 (2015), 599-614.

[36] Subuhi Khan, Ghazala Yasmin and Naeem Ahmad, On a new family related to truncated exponential and Sheffer polynomials, J. Math. Anal. Appl. 418 (2014), 921-937.

[37] H. L. Krall and O. Frink, A new class of orthogonal polynomials: the Bessel polynomials, Trans. Amer. Math. Soc. 65 (1949), 100-115.

[38] M. Lahiri, On a generalization of Hermite polynomials, Proc. Amer. Math. Soc. 27 (1971), $117-121$. 
[39] E. D. Rainville, Special Functions, Reprint of 1960 first edition. Chelsea Publishig Co., Bronx, New York, 1971.

[40] S. Roman, The Umbral Calculus, Academic Press, New York, 1984.

[41] J. F. Steffensen, The poweroid, an extension of the mathematical notion of power, Acta Mathematica 73 (1941), 333-366.

[42] G. Szegö, Orthogonal Polynomials, American Mathematical Society, Providence, RI, 1978. 